\title{
A systematic methodology for controller tuning in wastewater treatment plants
}

\author{
Mauricio Iglesias, Miguel; Jørgensen, Sten Bay; Sin, Gürkan
}

Publication date:

2012

Document Version

Publisher's PDF, also known as Version of record

Link back to DTU Orbit

Citation (APA):

Mauricio Iglesias, M., Jørgensen, S. B., \& Sin, G. (2012). A systematic methodology for controller tuning in wastewater treatment plants. Poster session presented at 8th IFAC Symposium on Advanced Control of Chemical Processes, Singapore, Singapore.

\section{General rights}

Copyright and moral rights for the publications made accessible in the public portal are retained by the authors and/or other copyright owners and it is a condition of accessing publications that users recognise and abide by the legal requirements associated with these rights.

- Users may download and print one copy of any publication from the public portal for the purpose of private study or research.

- You may not further distribute the material or use it for any profit-making activity or commercial gain

- You may freely distribute the URL identifying the publication in the public portal

If you believe that this document breaches copyright please contact us providing details, and we will remove access to the work immediately and investigate your claim. 


\section{Controller tuning in wastewater treatment plants}

Miguel Mauricio-Iglesias Sten Bay J ørgensen Gürkan Sin

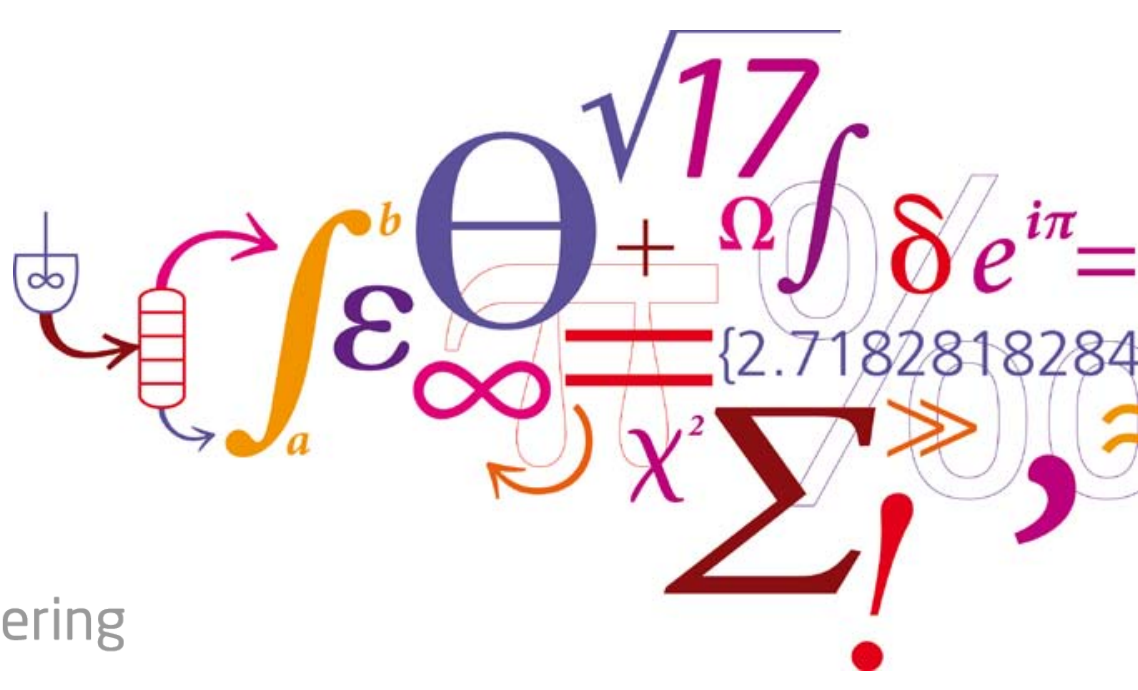




\section{Introduction and motivation}

Tuning a control loop is the adjustment of its control parameters to the optimum values for the desired control response

There is a large number of methods that can be used for tuning.

O'Dwyer, A. Handbook of Controller Tuning Rules

Åström, K. J. and Hägglund, T. PID Controllers: Theory, Design and Tuning

Wastewater treatment plants (WWTP) can be tuned according to any of those methods. They use mostly PI controllers

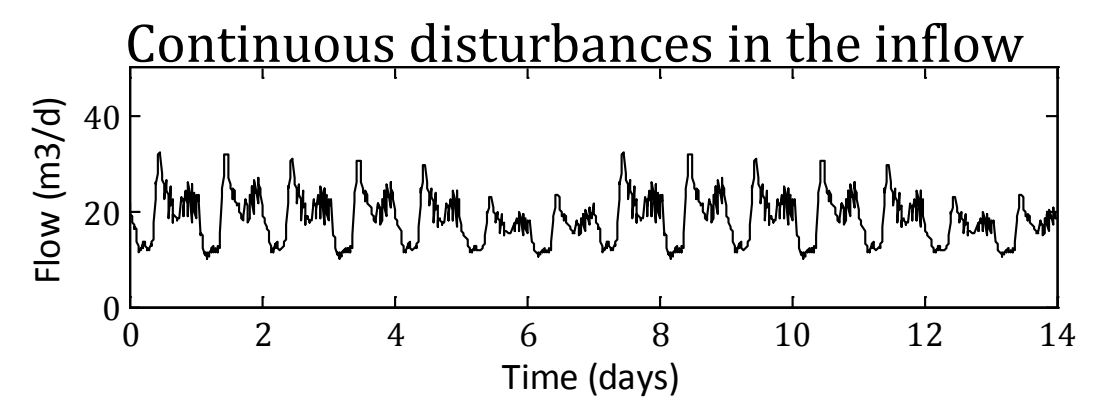

The purpose of this contribution is to use process engineering knowledge of WWTP and its influent dynamics to improve the tuning of the controllers! 


\section{Overview of the presentation}

1) Presentation of the WWTP

2) Methodology. Example

3) Process review

4) Open loop analysis

5) Closed loop design

6) Evaluation

7) Conclusion 


\section{Methodology}

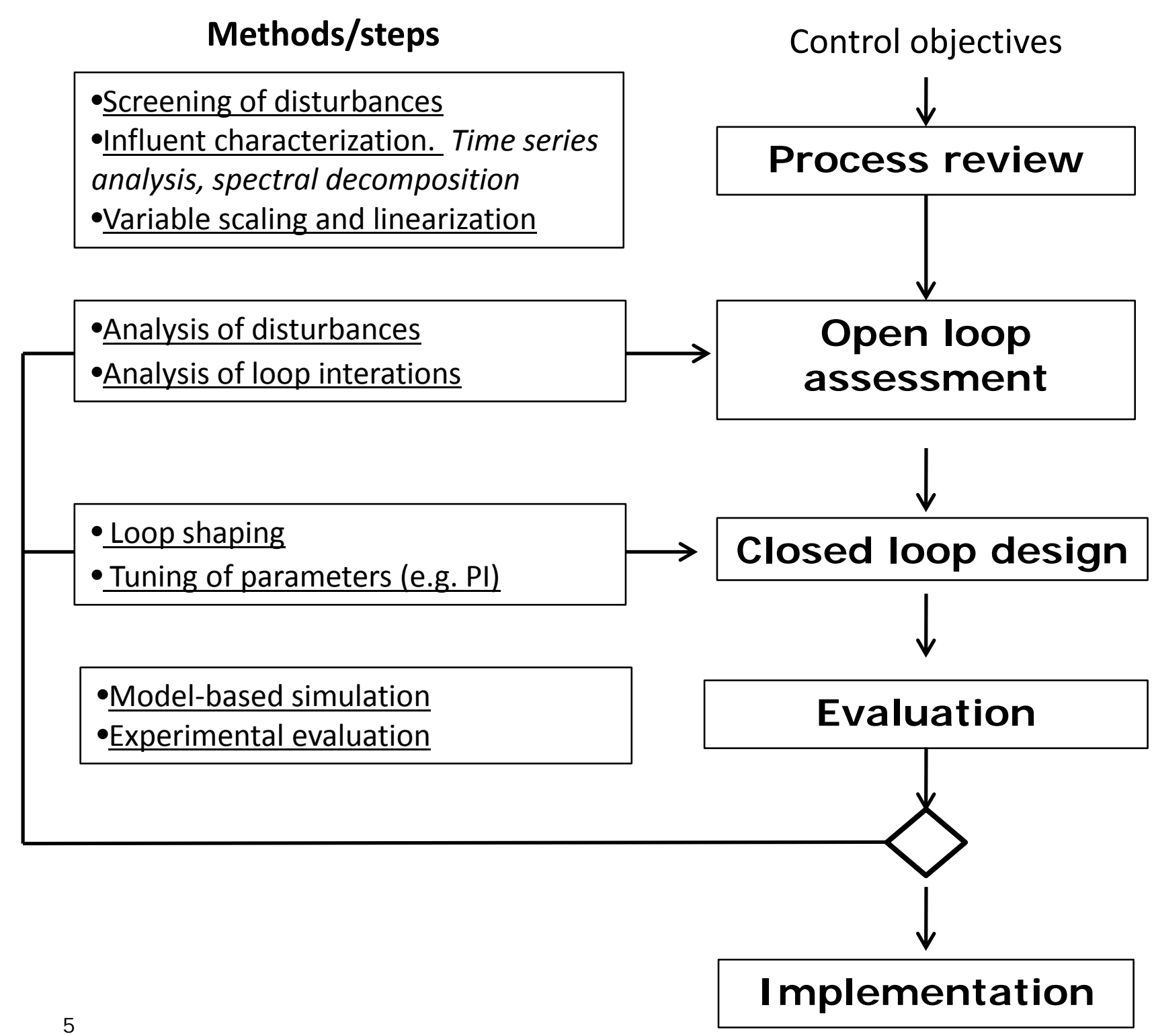




\section{Methodology}

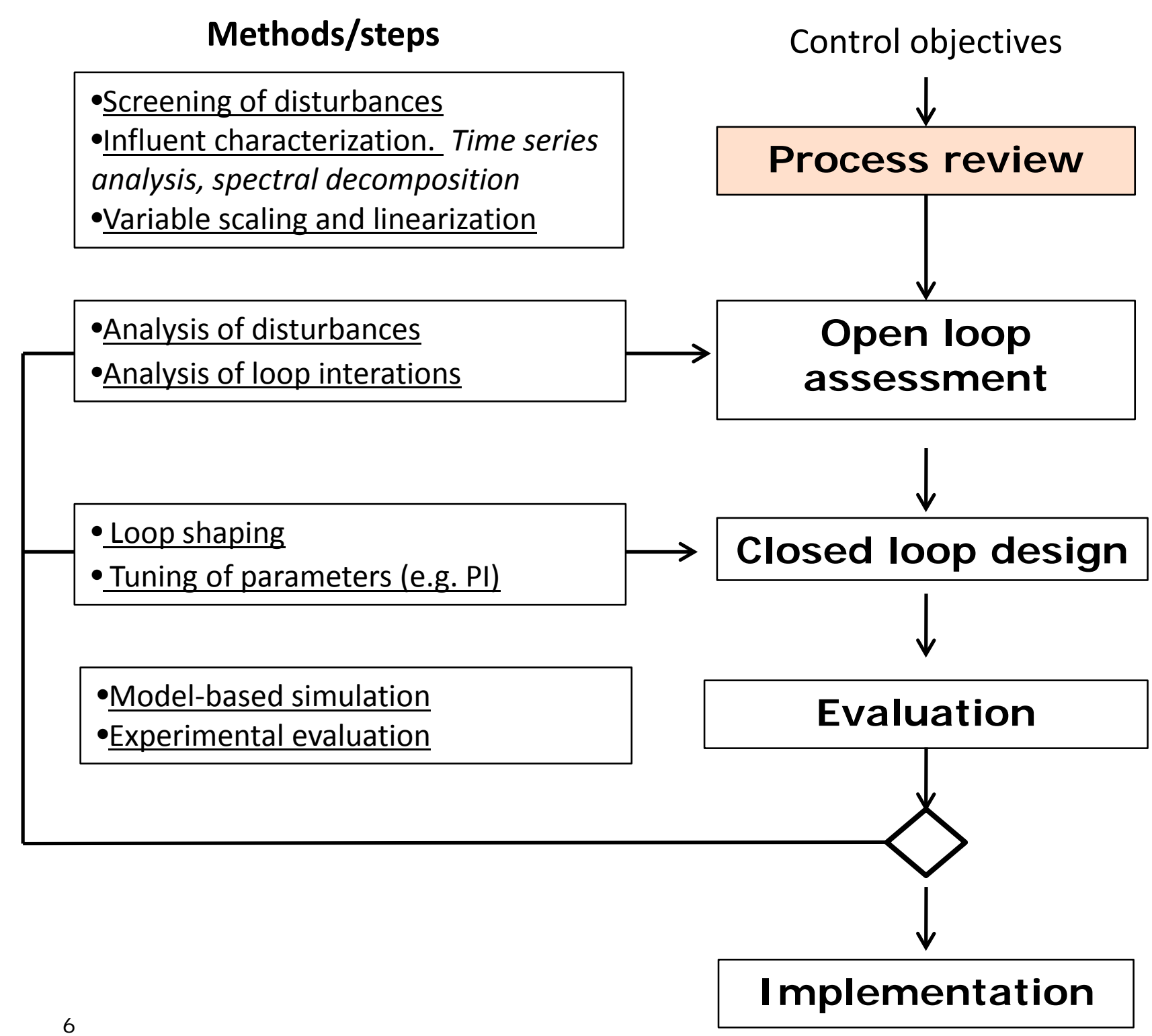




\section{Process review. Screening of disturbances}

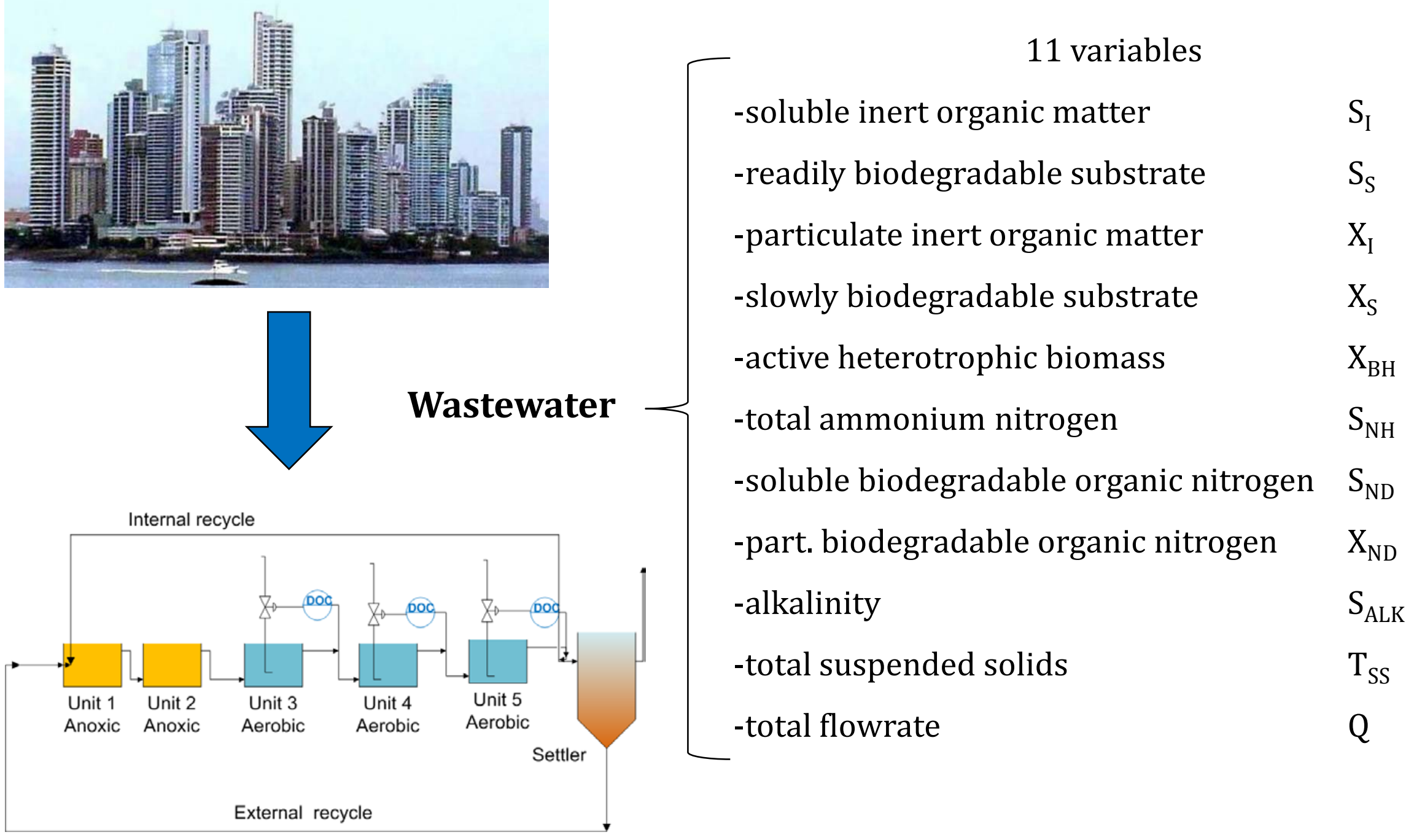




\section{Process review. Screening of disturbances}

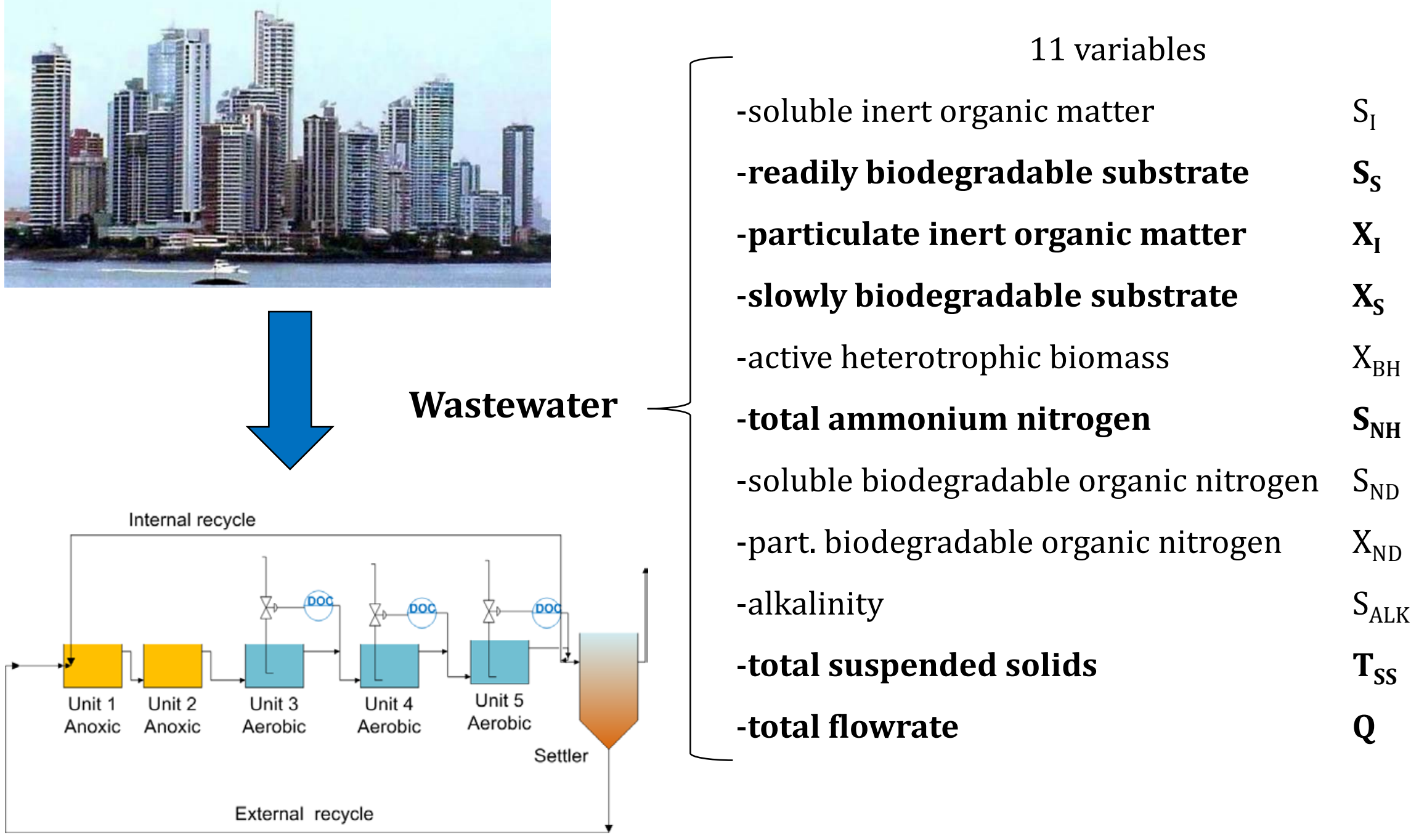




\section{Process review. Influent characterization \& dynamics}

\section{Dry weather}

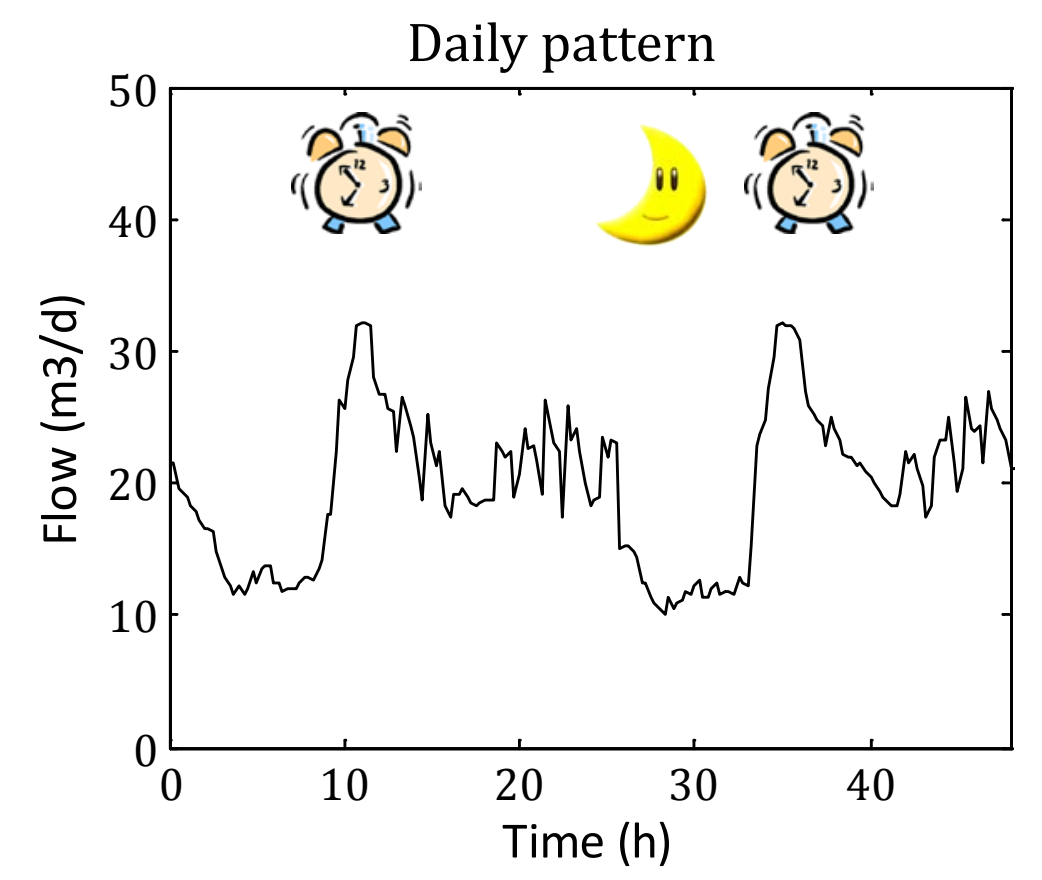

Weekly pattern

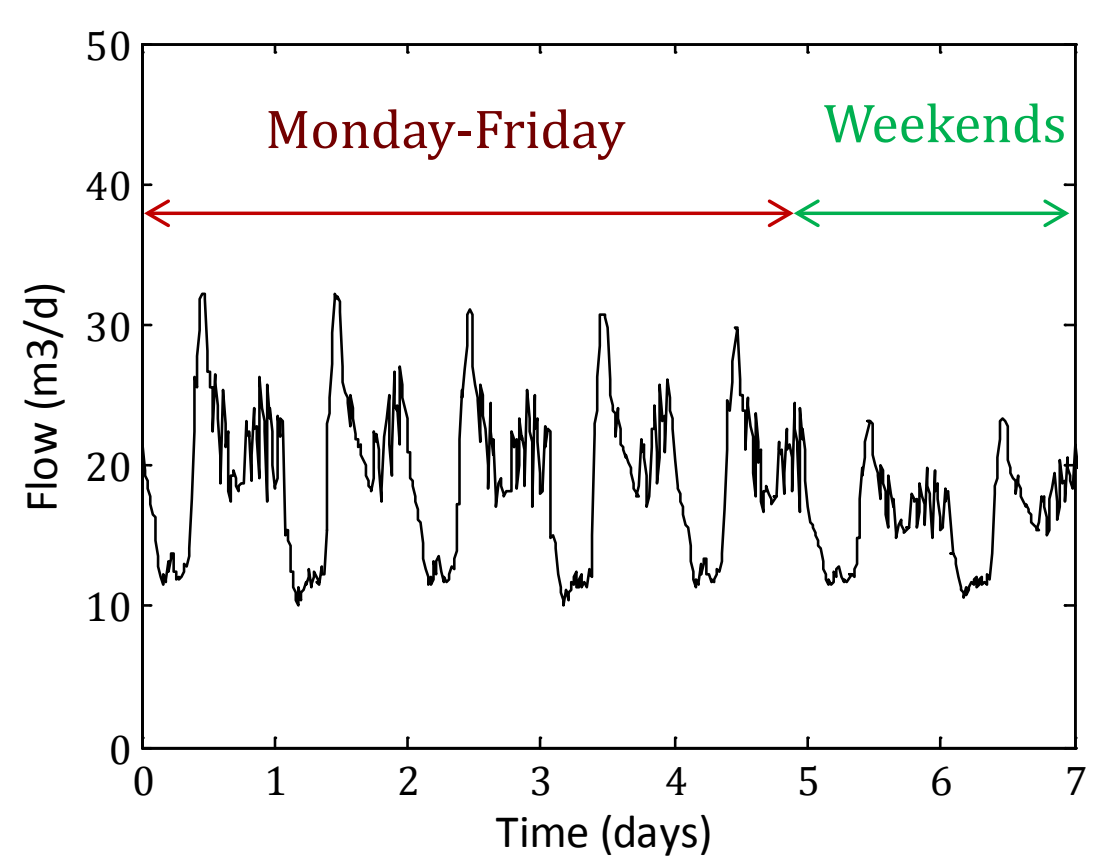




\section{Process review. Influent characterization}

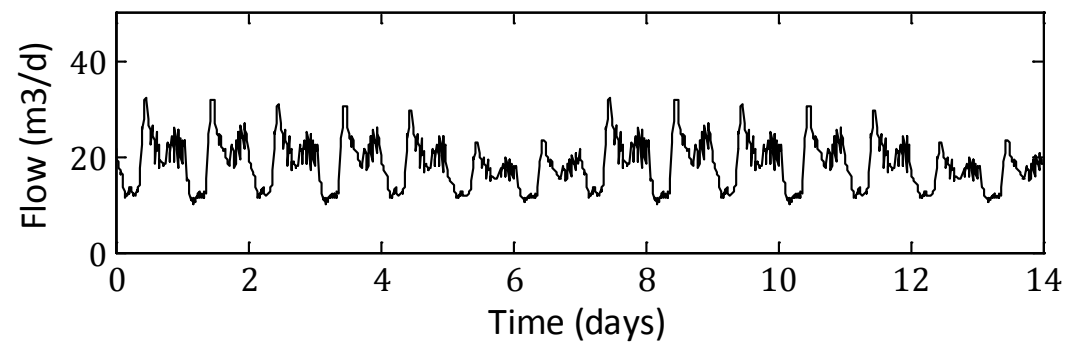

Spectral decomposition
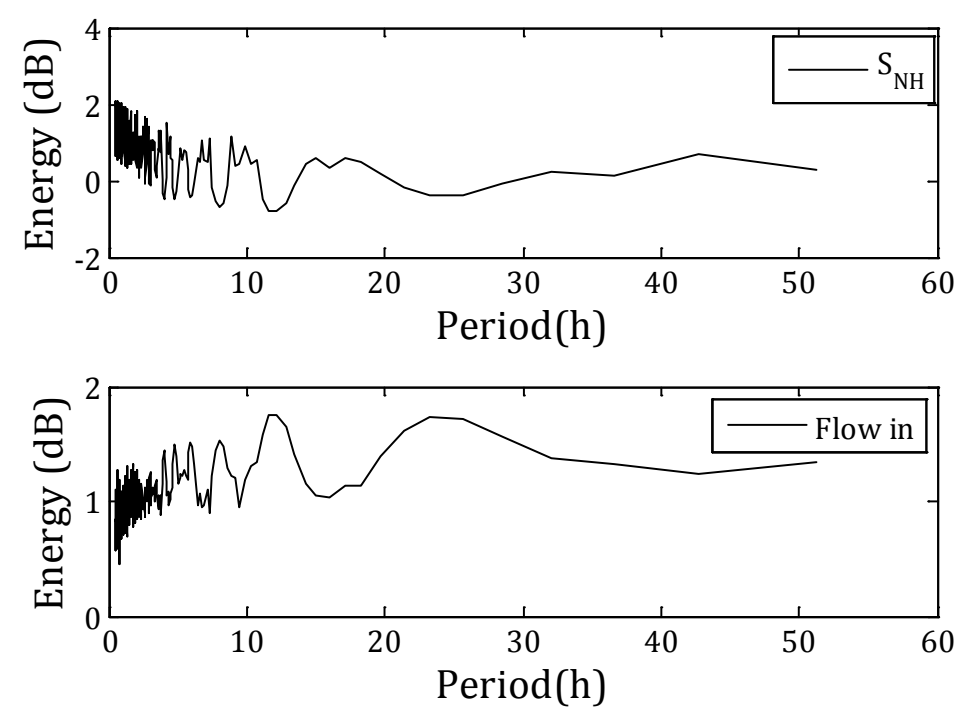

Main periods associated with influent dynamics

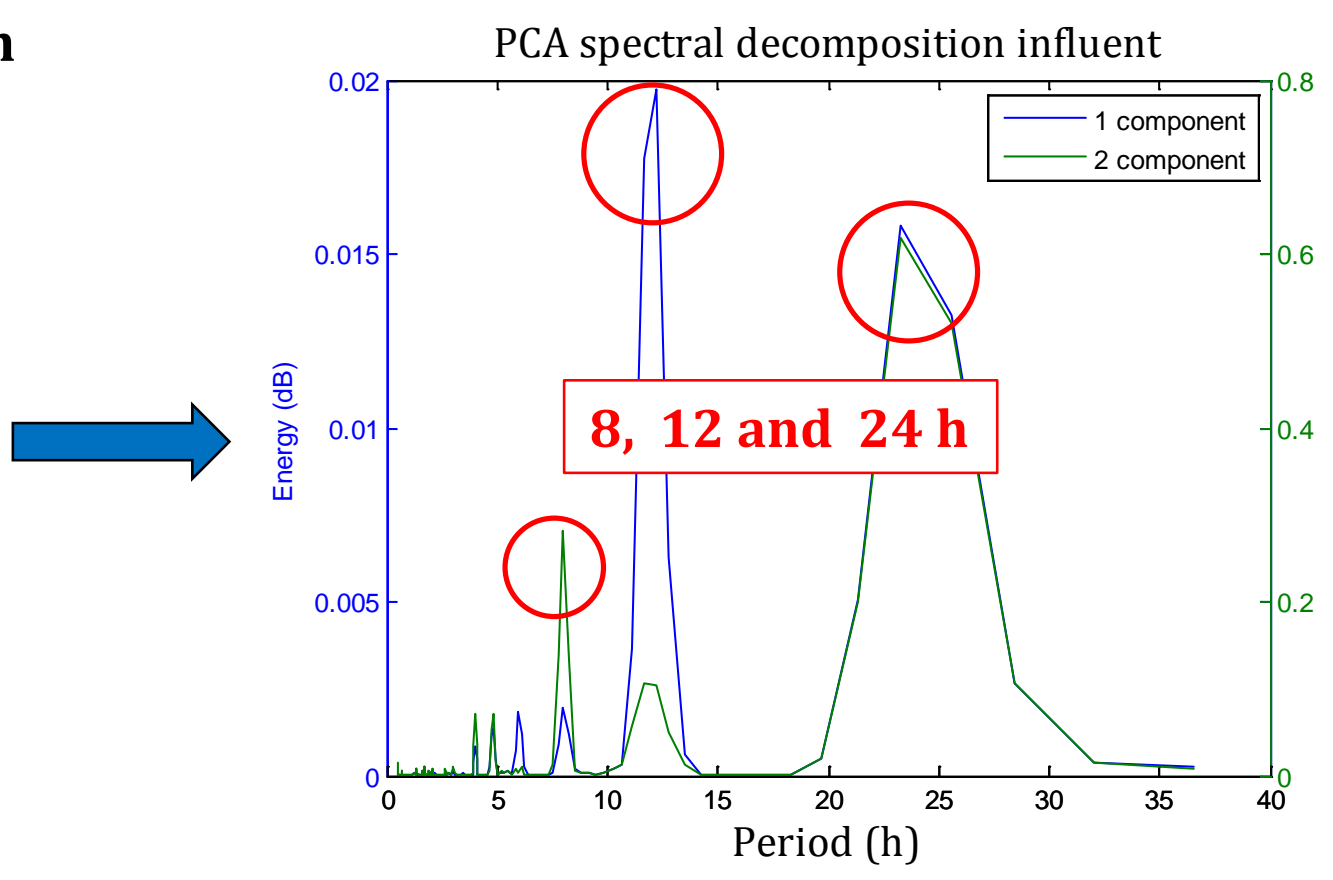

Principal component analysis 


\section{Process review. Variable scaling}

A number of criteria used depend critically on the scaling

\begin{tabular}{|c|c|c|c|c|}
\hline & $\begin{array}{l}\text { Var. } \\
\text { Value }\end{array}$ & $\begin{array}{l}\mathrm{S}_{\mathrm{I}}\left(\mathrm{g} \mathrm{COD} \mathrm{m}^{-3}\right) \\
30\end{array}$ & $\begin{array}{l}\mathrm{S}_{\mathrm{S}}\left(\mathrm{g} \mathrm{COD} \mathrm{m}^{-3}\right) \\
65.2\end{array}$ & $\begin{array}{l}\mathrm{X}_{\mathrm{I}}\left(\mathrm{g} \mathrm{COD} \mathrm{m}^{-3}\right) \\
45.6\end{array}$ \\
\hline \multirow{2}{*}{$\begin{array}{l}\text { Disturbances } \\
\left(d_{\max }-d_{\min }\right)\end{array}$} & Var. & $\mathrm{X}_{\mathrm{S}}\left(\mathrm{g} \mathrm{COD} \mathrm{m}^{-3}\right)$ & $\mathrm{X}_{\mathrm{BH}}\left(\mathrm{g} \mathrm{COD} \mathrm{m}^{-3}\right)$ & $\mathrm{S}_{\mathrm{NH}}\left(\mathrm{g} \mathrm{N} \mathrm{m}^{-3}\right)$ \\
\hline & Value & 193 & 26.5 & 30.1 \\
\hline \multirow{4}{*}{$\begin{array}{l}\text { for dry weather, rain } \\
\text { and storm weather }\end{array}$} & Var. & $S_{N D}\left(\mathrm{~g} \mathrm{~N} \mathrm{~m}^{-3}\right)$ & $\mathrm{X}_{\mathrm{ND}}\left(\mathrm{g} \mathrm{N} \mathrm{m}^{-3}\right)$ & $\mathrm{S}_{\mathrm{ALK}}\left(\mathrm{mol} \mathrm{m}^{-3}\right)$ \\
\hline & Value & 6.5 & 10 & 7 \\
\hline & Var. & $\mathrm{T}_{\mathrm{SS}}\left(\mathrm{gCOD} \mathrm{m}^{-3}\right)$ & $Q\left(m^{3} d^{-1}\right)$ & \\
\hline & Value & 199 & 18446 & \\
\hline \multirow{3}{*}{ CVs $\Delta y_{\max }$} & Var. & $\mathrm{D}_{03}\left(\mathrm{gO} \mathrm{O} \mathrm{m}^{-3}\right)$ & $\mathrm{D}_{04}\left(\mathrm{~g} \mathrm{O} \mathrm{m}^{-3}\right)$ & $\mathrm{D}_{05}\left(\mathrm{gO} \mathrm{O} \mathrm{m}^{-3}\right)$ \\
\hline & Value & 0.1 & 0.1 & 0.1 \\
\hline & Var. & $\mathrm{kLa}_{3}\left(\mathrm{~d}^{-1}\right)$ & $\mathrm{kLa}_{4}\left(\mathrm{~d}^{-1}\right)$ & $\mathrm{kLa}_{5}\left(\mathrm{~d}^{-1}\right)$ \\
\hline MVs & Value & 360 & 360 & 360 \\
\hline
\end{tabular}




\section{Process review. Plant linearisation}

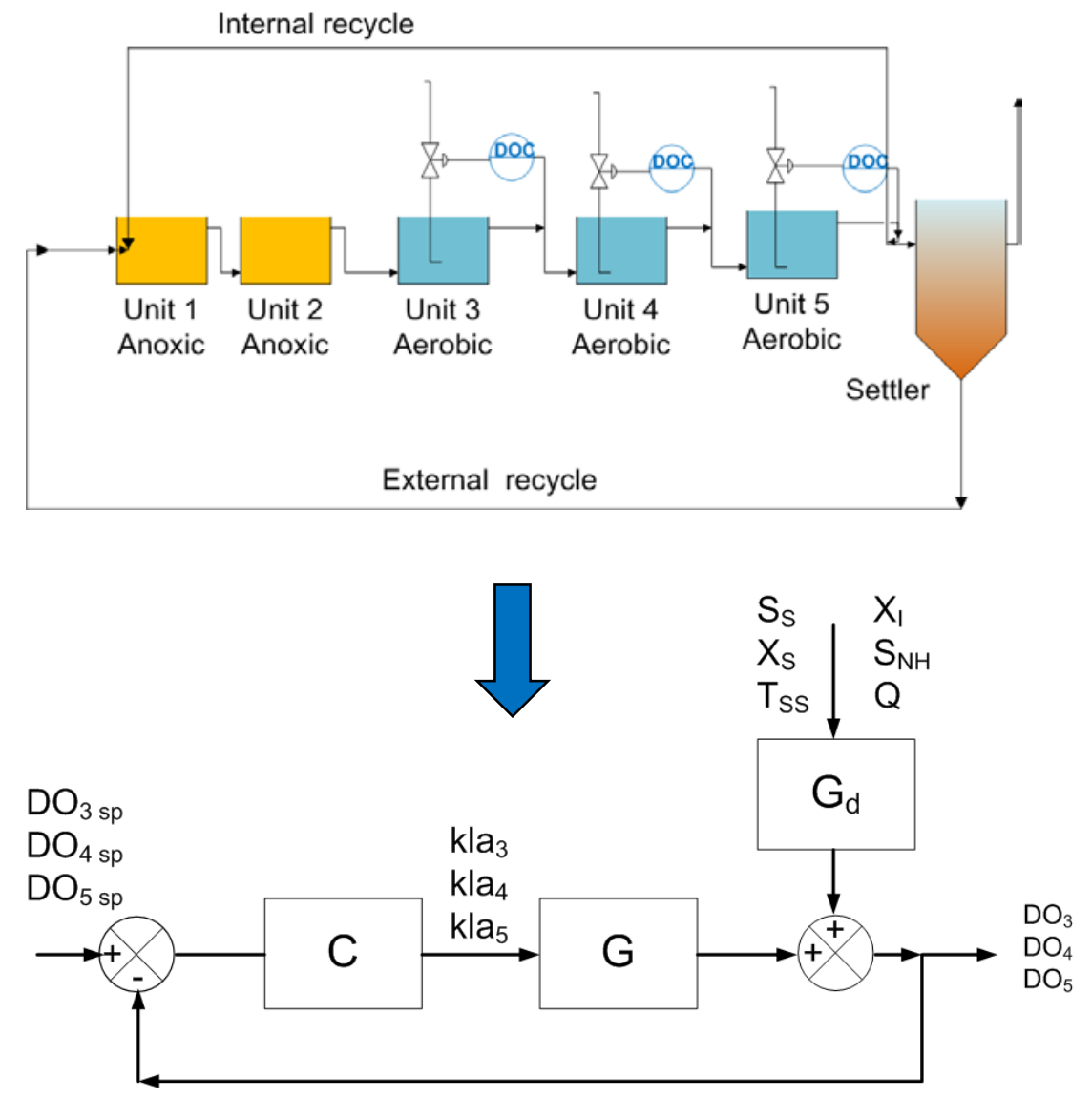

Full linear model (96 states)

Reduced linear model (1 state)
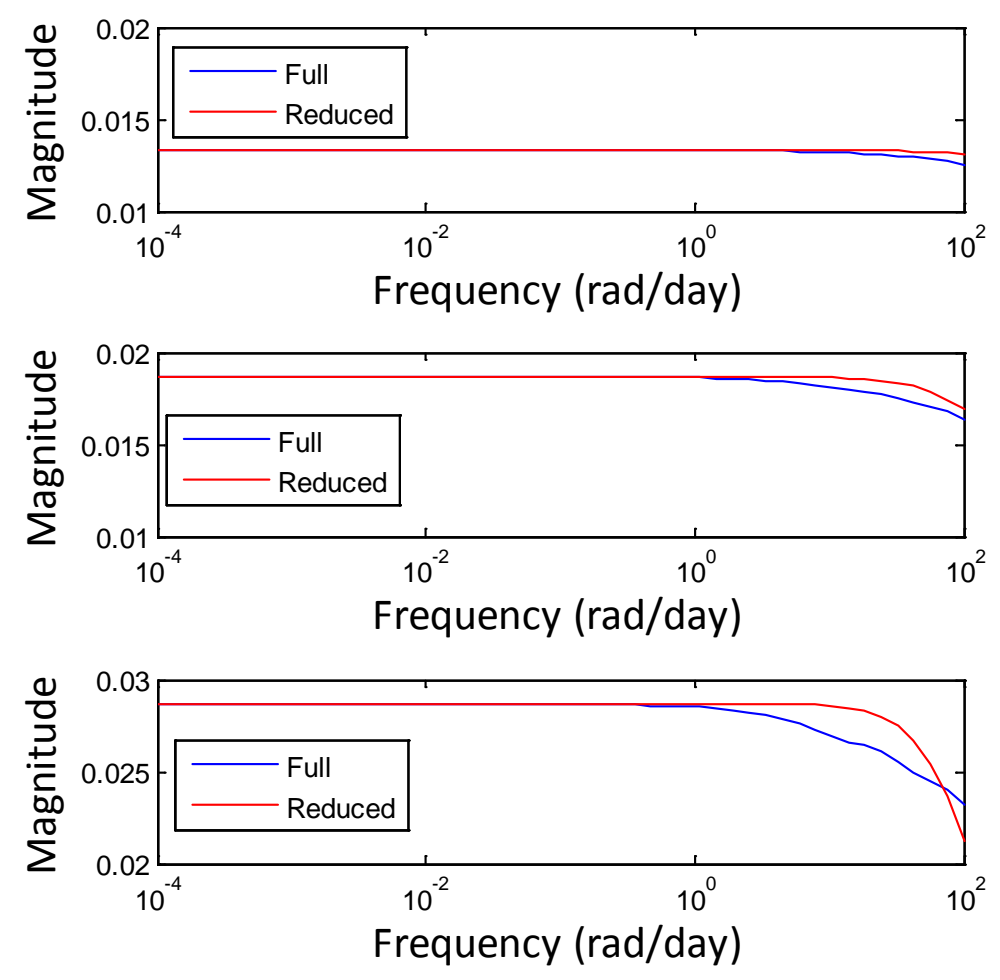


\section{Methodology}

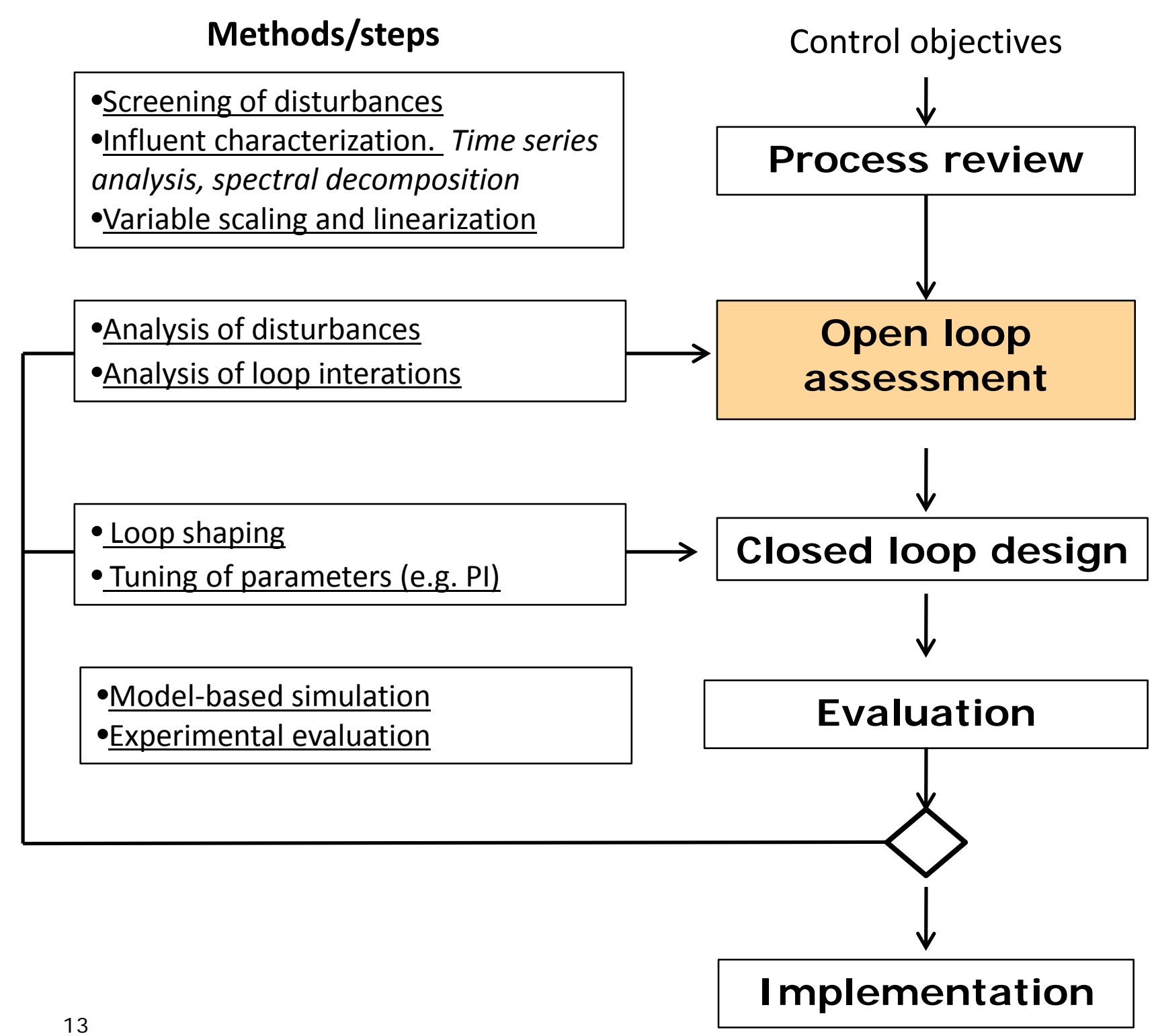




\section{Open loop assessment. Disturbances}

\section{Closed Loop Disturbance Gain (CLDG)}

$$
\begin{gathered}
C D L G=\tilde{G}_{(s)} G^{-1}{ }_{(s)} G_{d}(s) \\
\text { CLDG }=\left|\delta_{\mathbf{i}}\right|<\mathbf{1}
\end{gathered}
$$

The disturbance effect is lower than $\Delta y_{\max }$

$$
\text { CLDG }=\left|\delta_{\mathbf{i}}\right|>\mathbf{1}
$$

The disturbance effect is higher than $\Delta y_{\max }$ Need of control action!

$$
\left|\mathbf{g}_{\mathbf{i}} \mathbf{c}_{\mathbf{i}}\right|>\left|\delta_{\mathrm{i}}\right|
$$
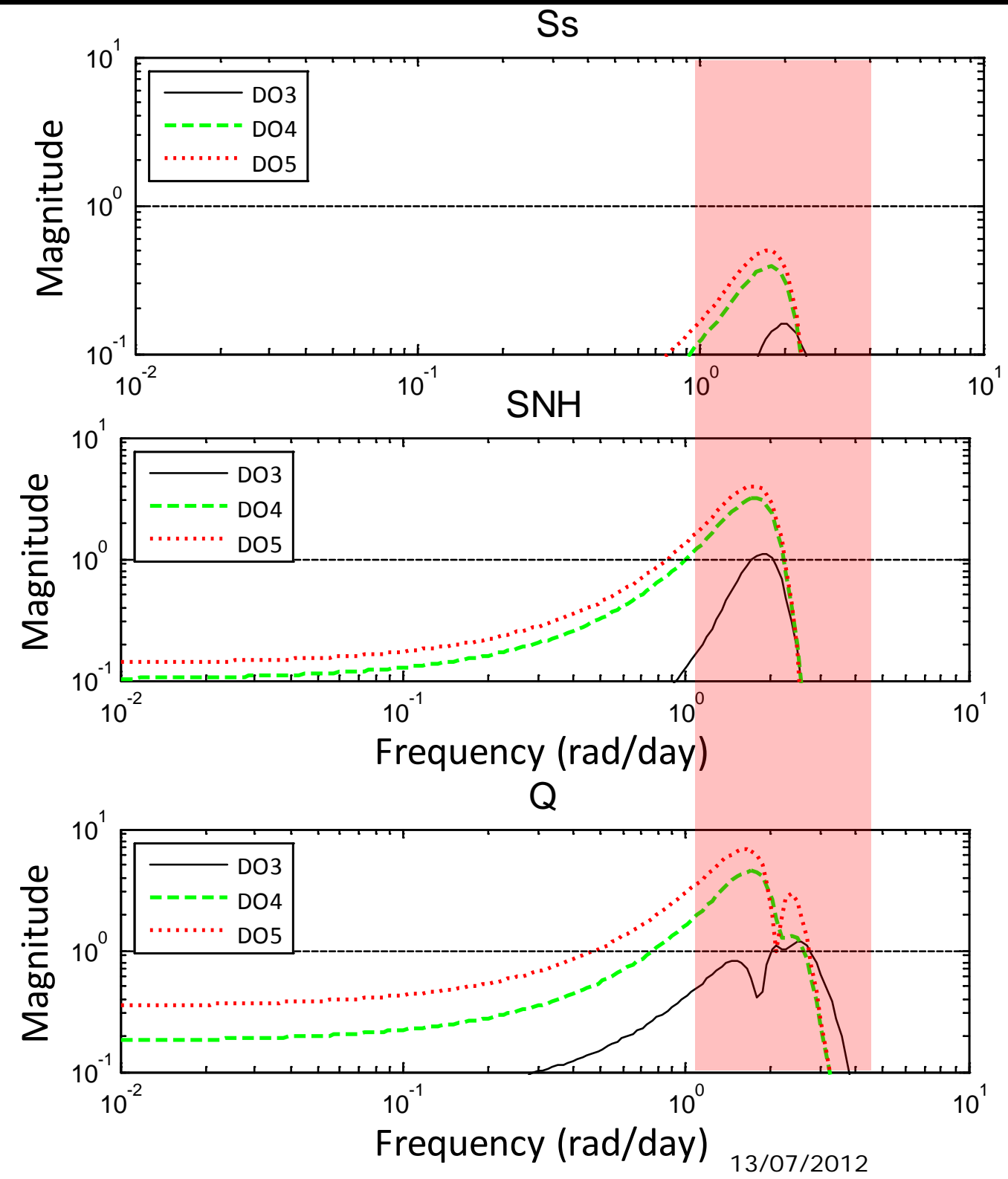


\section{Open loop assessment. Interactions}

Relative Gain Array (RGA)

$$
\lambda_{i j}=\frac{\left.\left(\partial y_{i} / \partial u_{j}\right)\right|_{u_{k}=0, k \neq j}}{\left.\left(\partial y_{i} / \partial u_{j}\right)\right|_{y_{k}=0, k \neq i}}
$$

$$
\lambda_{i j}=\frac{\text { Open loop gain }}{\text { Closed loop gain }}
$$
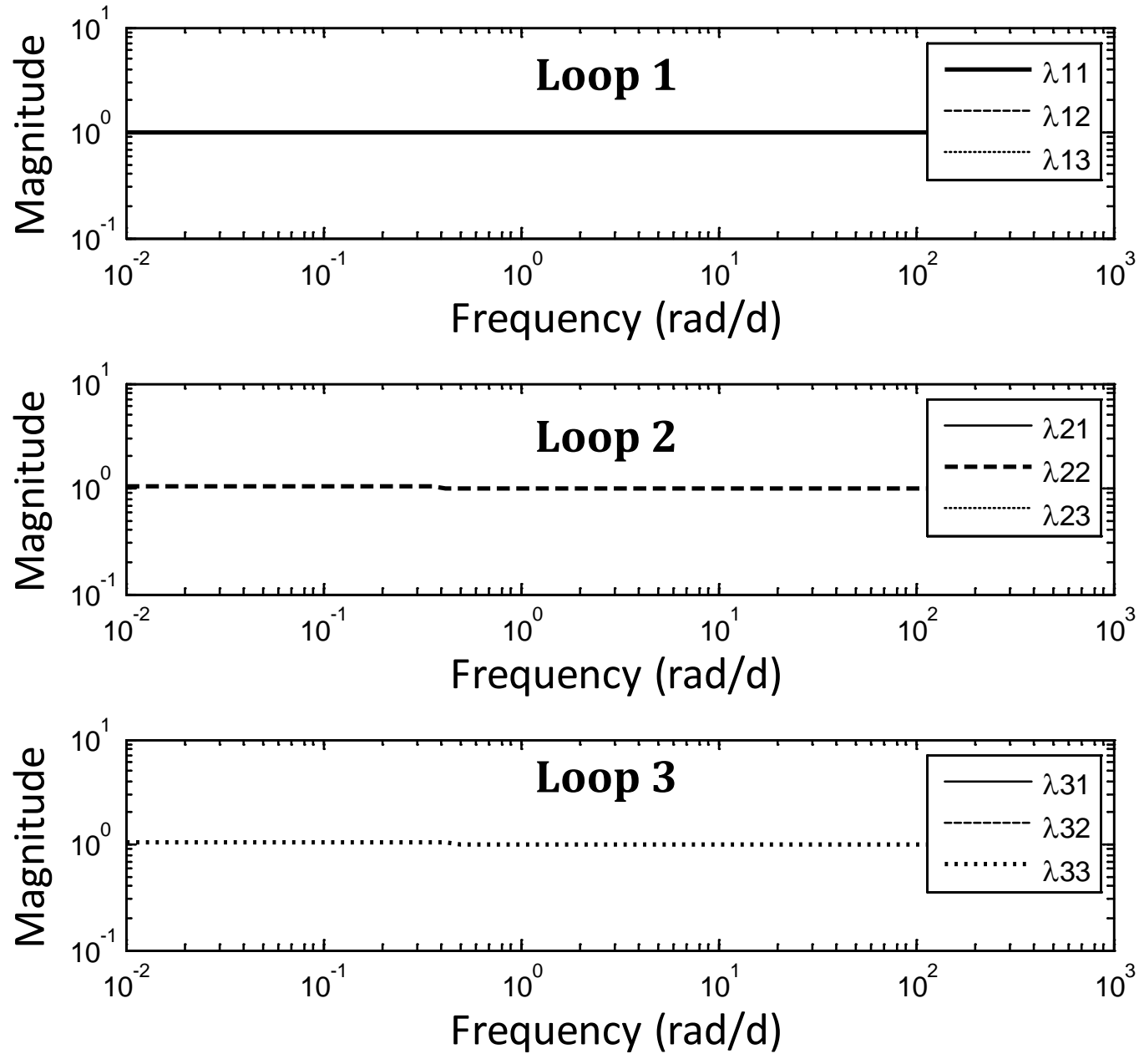


\section{Process review. Plant linearisation}

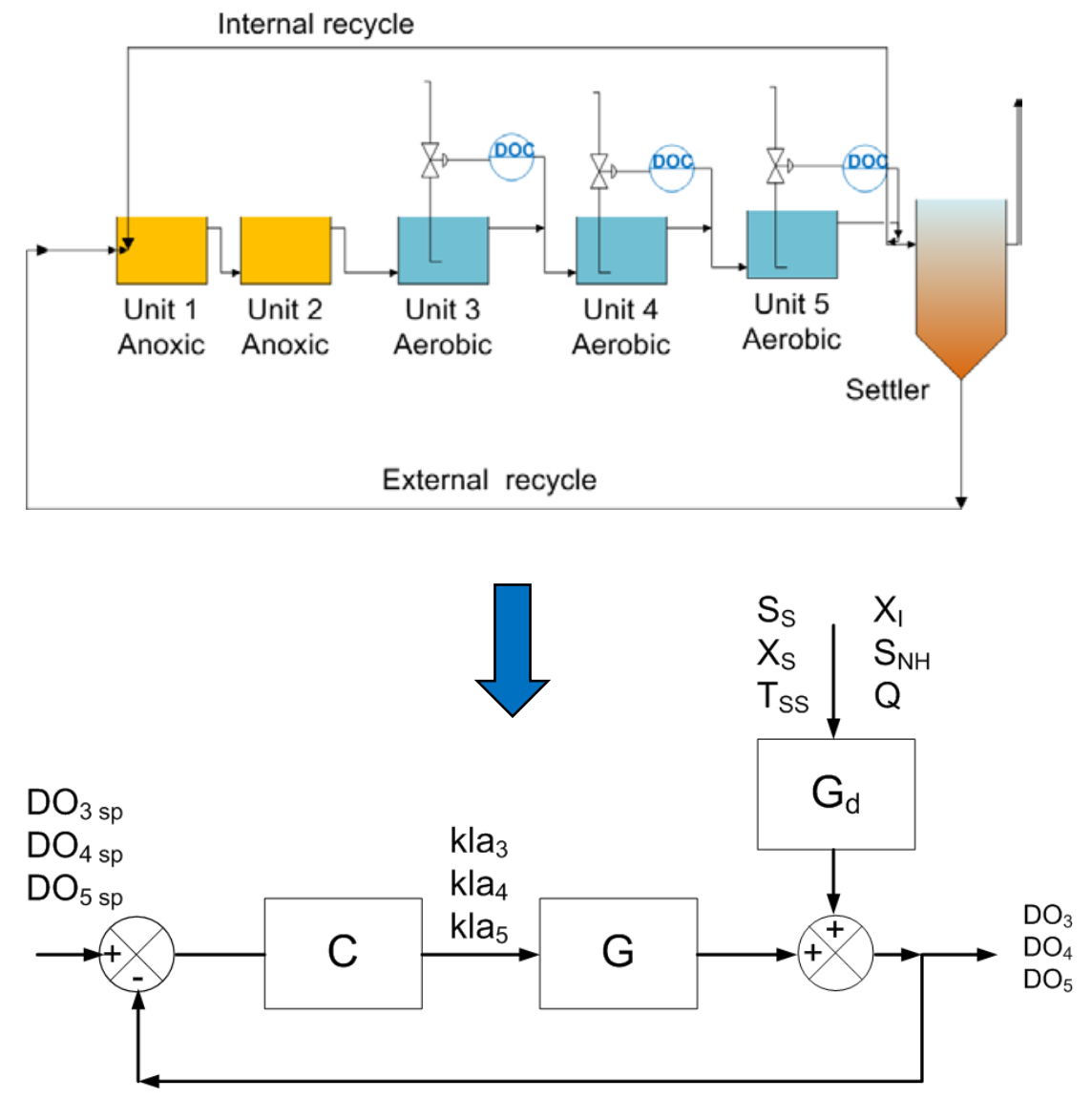

Full linear model (96 states)

Reduced linear model (1 state)
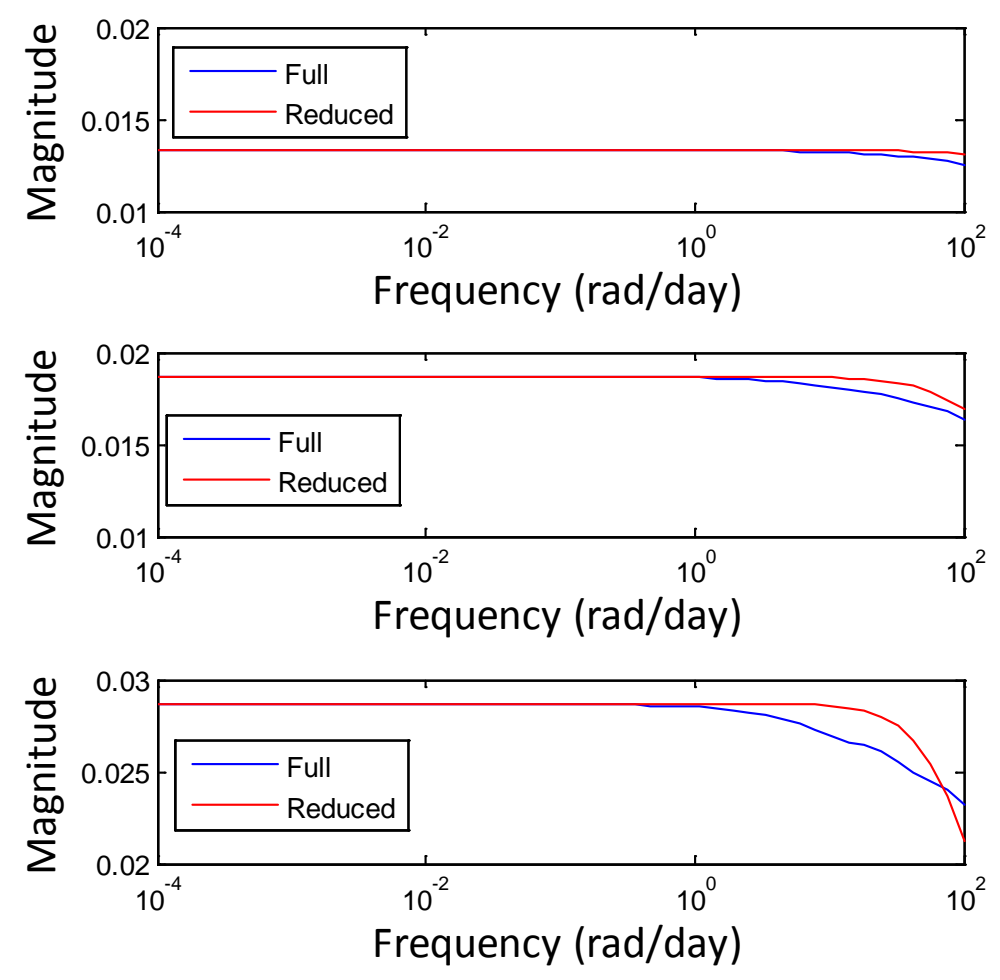


\section{Open loop assessment. Interactions}

Performance Relative Gain Array (PRGA)

$$
P R G A=\Gamma=\tilde{G}_{(s)} G^{-1}(s)
$$

9 Dependent on scaling

Suitable for one-way interactions
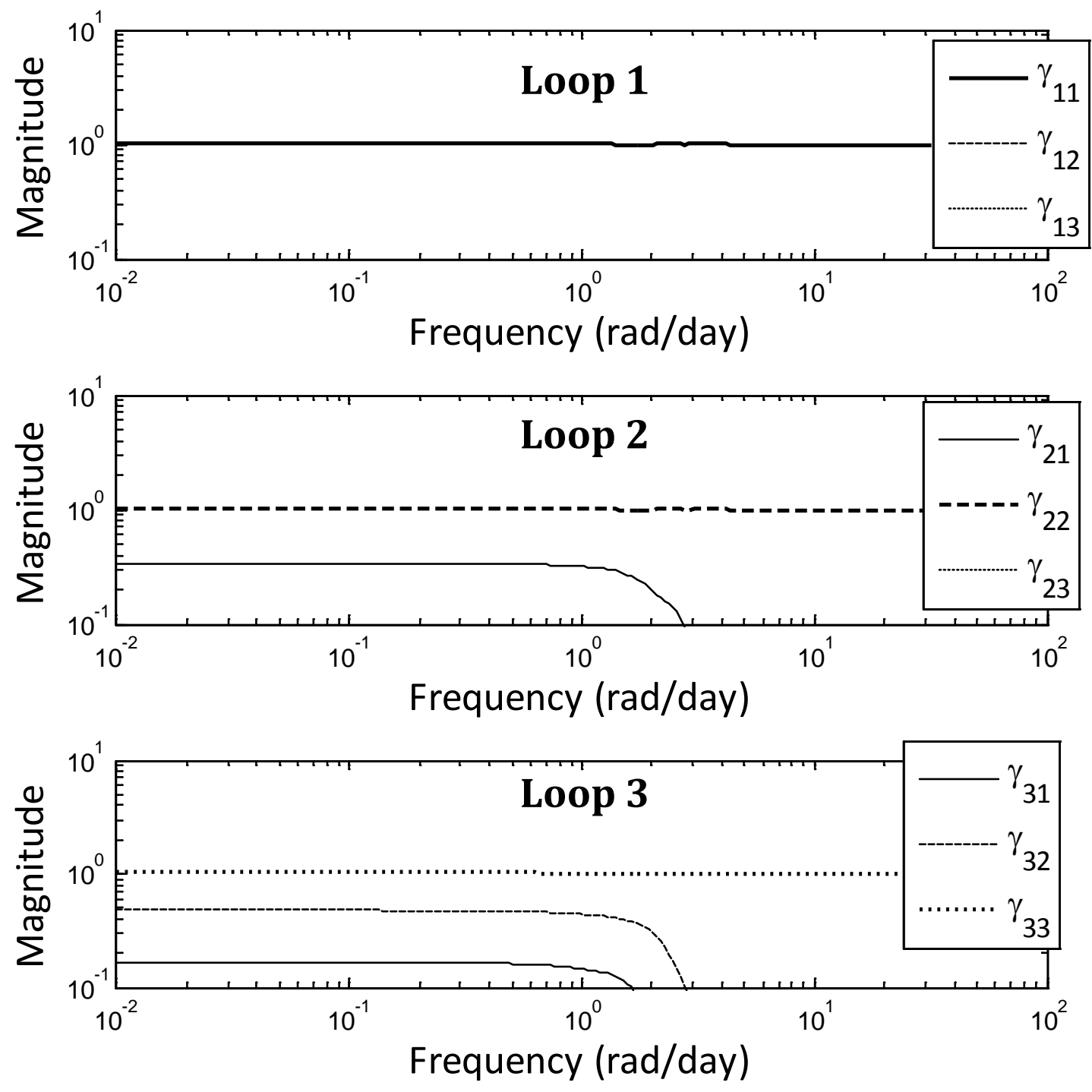

$13 / 07 / 2012$ 


\section{Methodology}

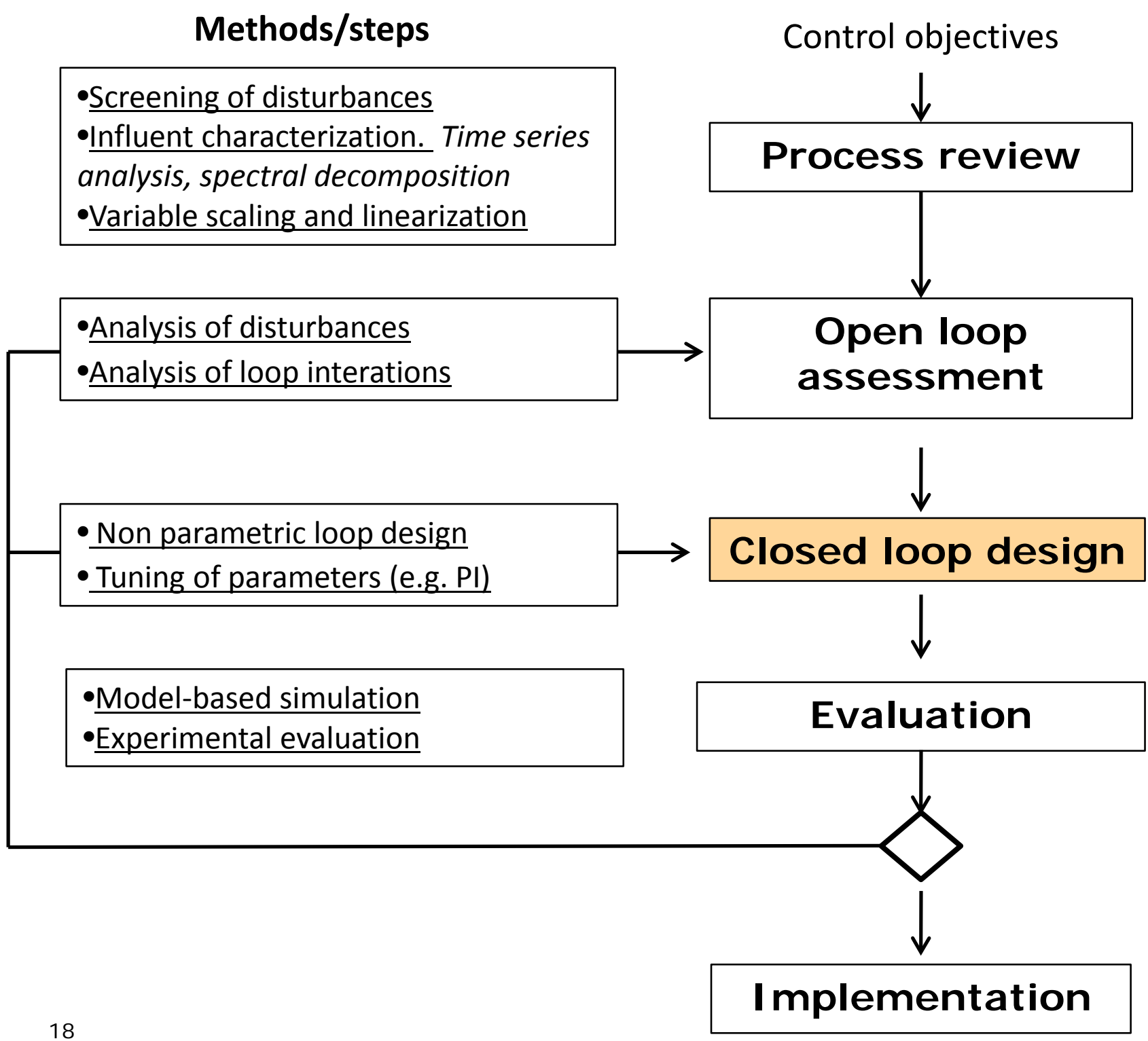




\section{Closed loop design. Analysis of tuning parameters}

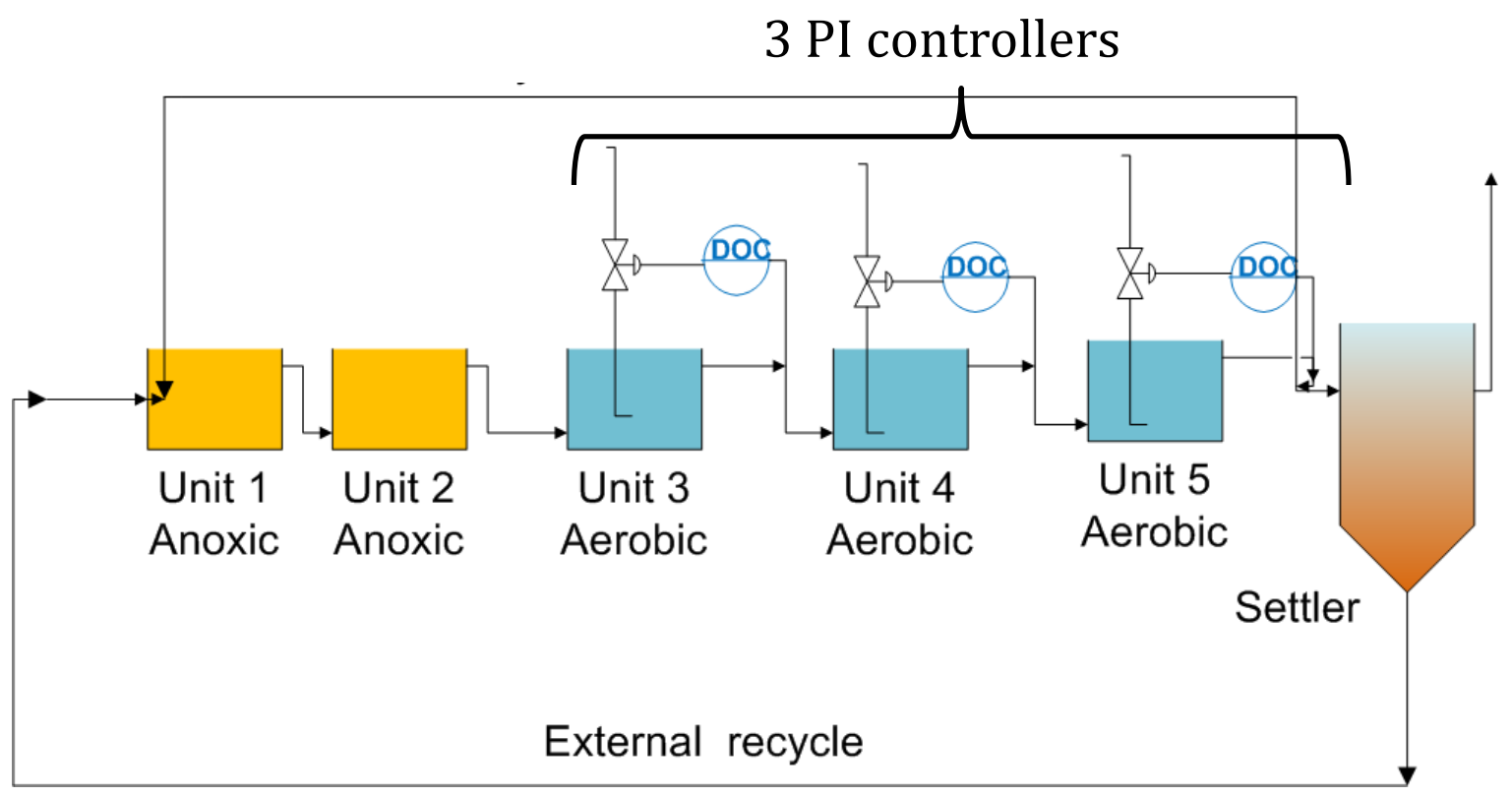

Reported parameters Vanrolleghem and Gillot 2002

$\begin{array}{lccc} & \text { Loop 1 } & \text { Loop 2 } & \text { Loop 3 } \\ \mathbf{K}_{\mathbf{p}} & 0.028 & 0.028 & 0.028 \\ \tau_{\mathbf{I}}(\min ) & 14.4 & 14.4 & 14.4\end{array}$




\section{Closed loop design. Analysis of tuning parameters}

$\mathbf{C L D G}=\left|\delta_{\mathrm{i}}\right|>\mathbf{1}$ at some frequencies

$$
\left|\mathrm{g}_{\mathrm{i}} \mathrm{c}_{\mathrm{i}}\right|>\left|\delta_{\mathrm{i}}\right| ?
$$

\section{CLDG for flow disturbance}
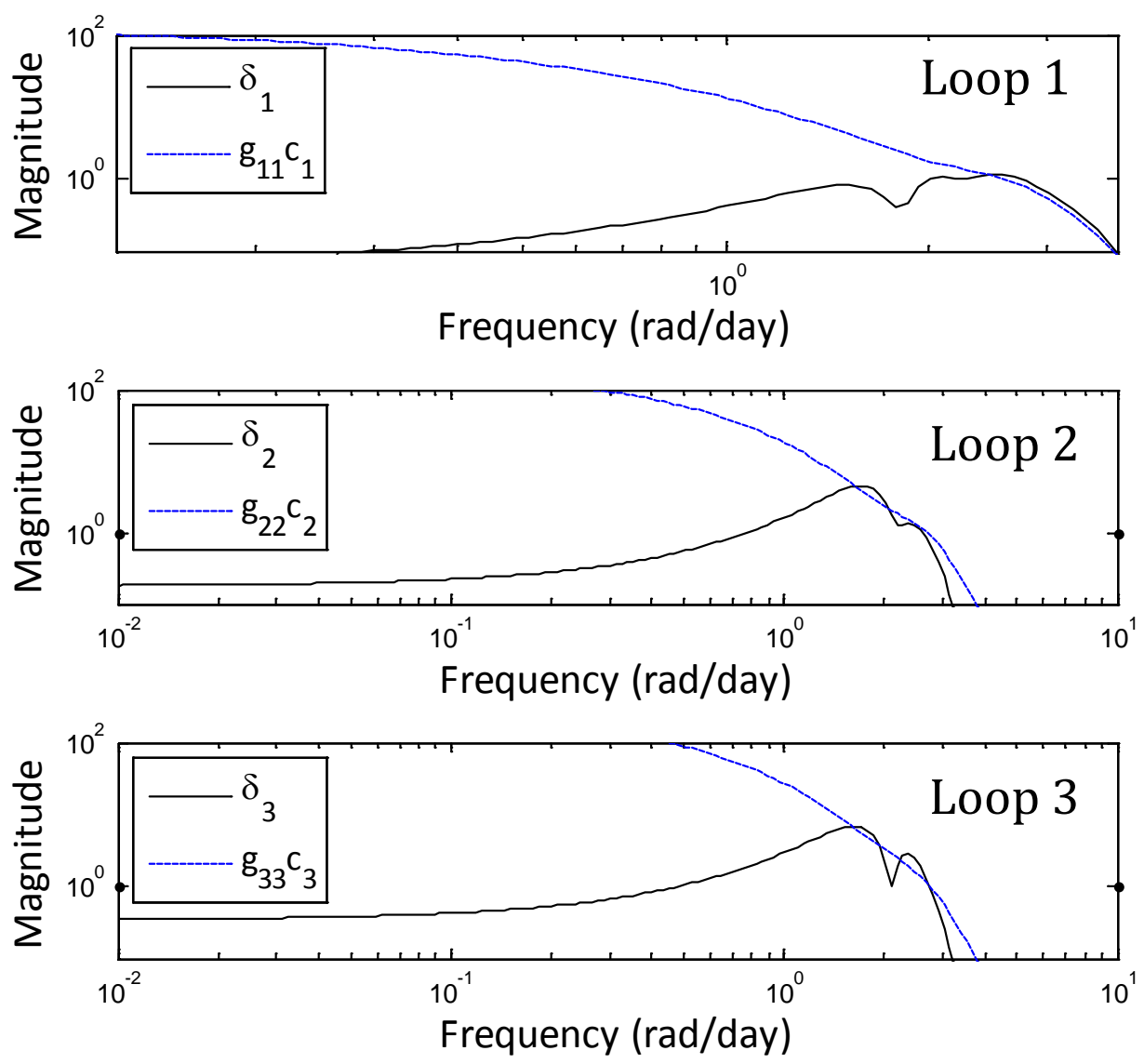


\section{Closed loop design. New tuning parameters}

\section{CLDG for flow disturbance}

$\min \left|M_{S}\right|_{\infty}=f\left(K_{p}, \tau_{I}\right)$

for $S(s)=\frac{I}{I+G(s) C(s)}$

s.t. $\left|g_{\mathrm{i}} \mathrm{c}_{\mathrm{i}}\right|>1.10\left|\delta_{\mathrm{i}}\right|$ for $\omega<\omega_{B}$

$\mathbf{K}_{\mathbf{p}}$

$\tau_{\mathrm{I}}(\mathrm{min})$

\begin{tabular}{ccc}
\multicolumn{3}{c}{ New parameters } \\
Loop 1 & Loop 2 & Loop 3 \\
0.056 & 0.056 & 0.056 \\
10.1 & 10.1 & 10.1
\end{tabular}
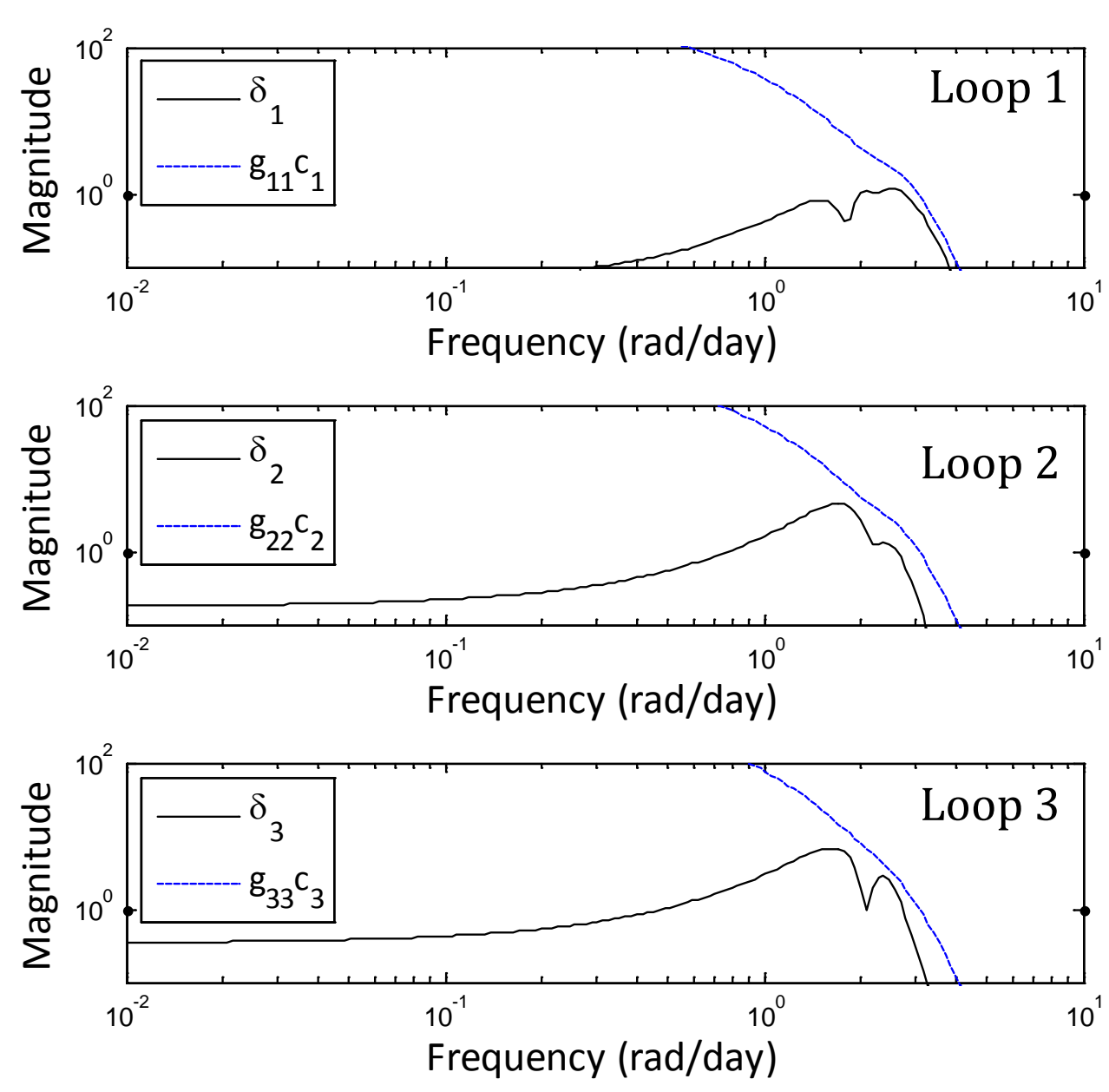


\section{Methodology}

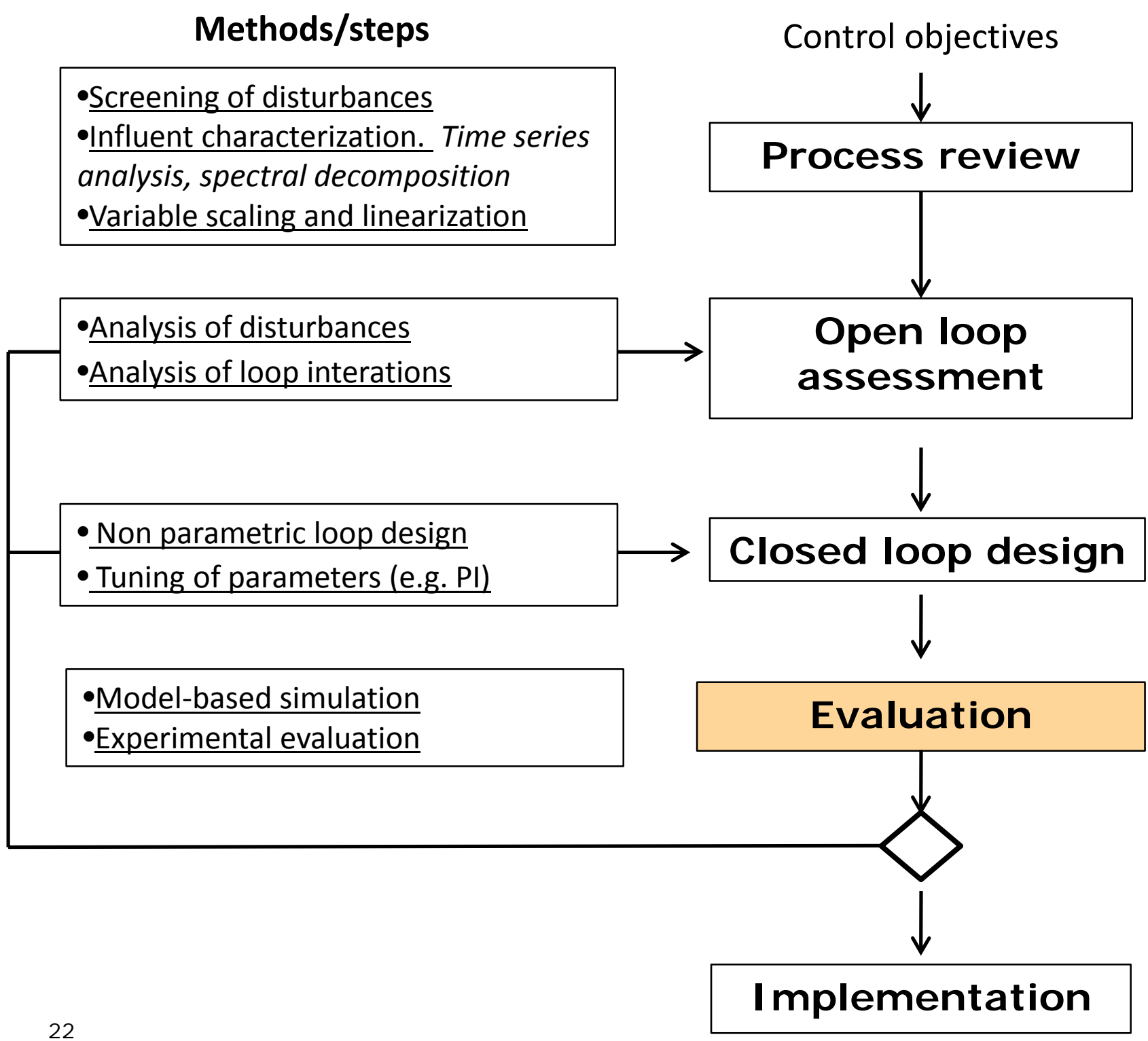




\section{Evaluation}

7 days dry weather +7 days storm weather

$$
\begin{aligned}
& I A E=\int_{0}^{\infty}\left|D O-D O^{s p}\right| d t \\
& T V=\sum_{i=1}^{\infty}\left|k l a_{i+1}-k l a_{i}\right|
\end{aligned}
$$

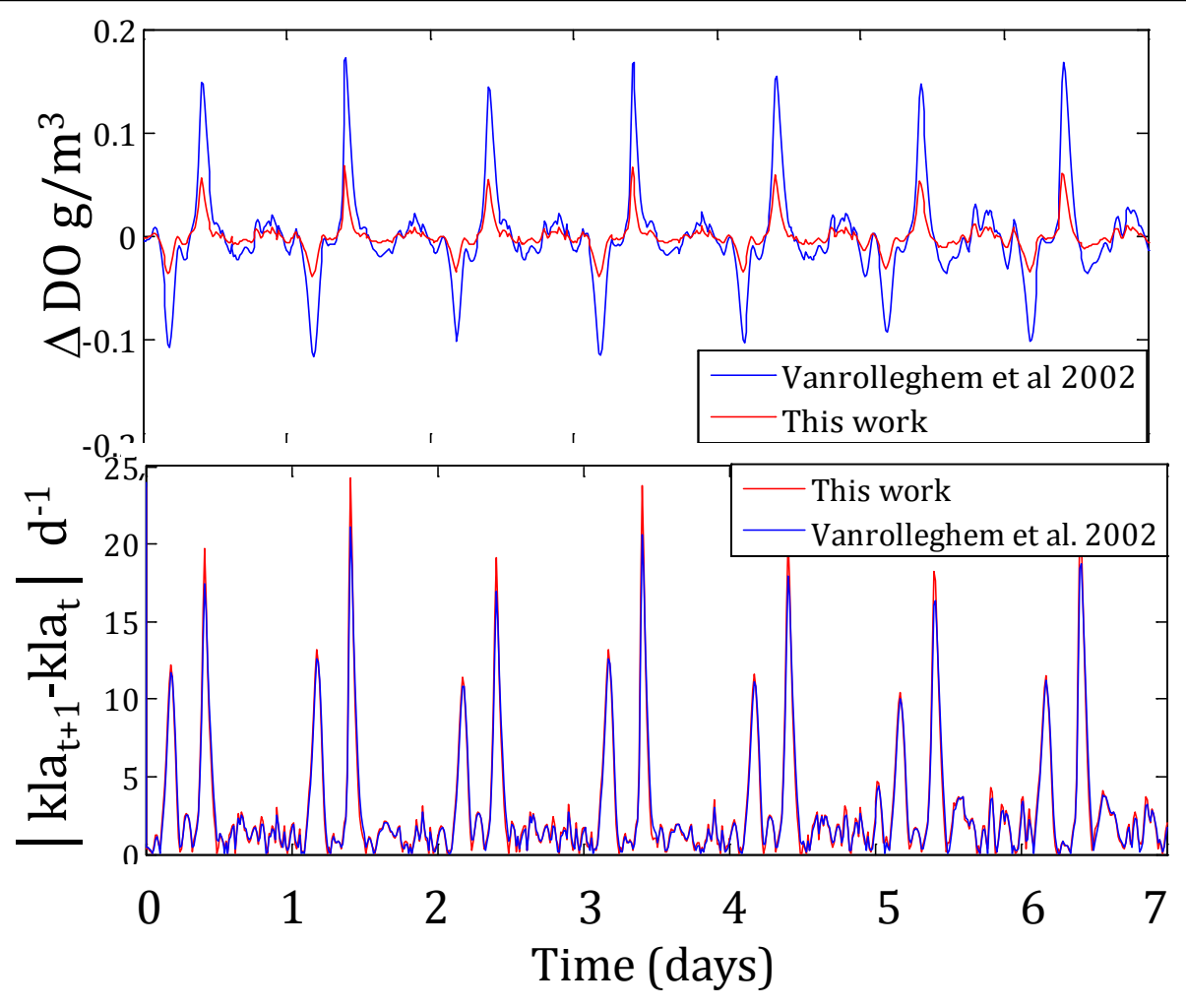




\section{Evaluation}

7 days dry weather +7 days storm weather

$$
\begin{aligned}
& I A E=\int_{0}^{\infty}\left|D O-D O^{s p}\right| d t \\
& T V=\sum_{i=1}^{\infty}\left|k l a_{i+1}-k l a_{i}\right|
\end{aligned}
$$

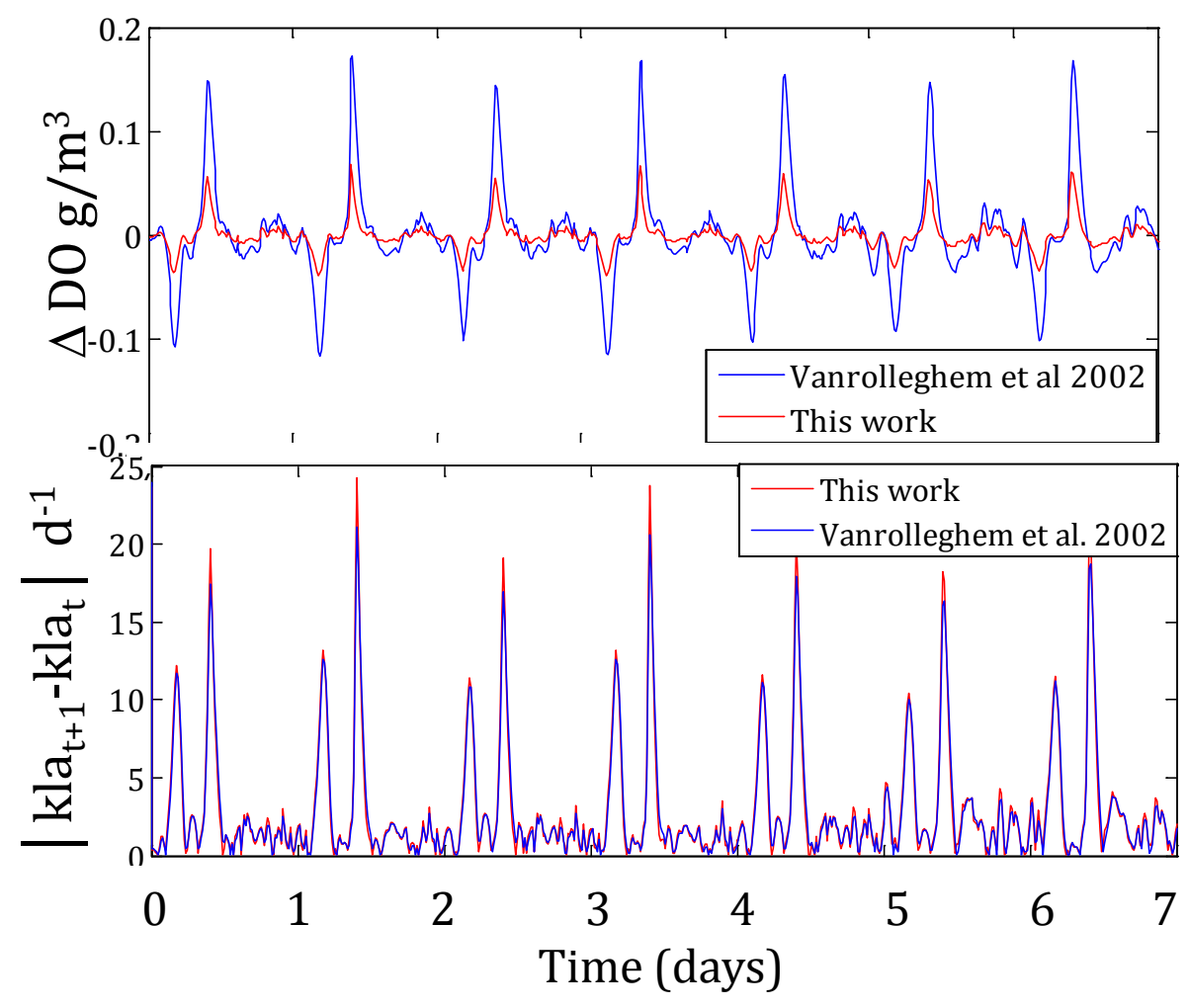

Integral of absolute error $\left(\mathrm{mgO}_{2} \mathrm{~d} \mathrm{~L}^{-1}\right)$

\begin{tabular}{|c|c|c|c|c|c|c|}
\hline & $\mathrm{DO}_{3}$ & $\mathrm{DO}_{4}$ & $\mathrm{DO}_{5}$ & $\mathbf{k L a}_{3}$ & $\mathbf{k L a}_{4}$ & $\mathbf{k L a}_{5}$ \\
\hline \multicolumn{7}{|c|}{ Dryweather influent } \\
\hline Vanrolleghem \&Gillot $_{(2002)}$ & 0.190 & 0.326 & 0.315 & 14.0 & 31.5 & 31.3 \\
\hline This work & 0.020 & 0.034 & 0.032 & 16.1 & 35.1 & 34.7 \\
\hline \multicolumn{7}{|c|}{ Storm weather influent } \\
\hline Vanrolleghem \&Gillot $_{(2002)}$ & 0.193 & 0.313 & 0.323 & 13.2 & 31.5 & 33.3 \\
\hline This work & 0.021 & 0.032 & 0.033 & 15.3 & 31.5 & 33.3 \\
\hline
\end{tabular}

Total Variance of MV $\left(\mathbf{d}^{-1}\right)$ 


\section{Evaluation}

7 days dry weather +7 days storm weather

$$
\begin{aligned}
& I A E=\int_{0}^{\infty}\left|D O-D O^{s p}\right| d t \\
& T V=\sum_{i=1}^{\infty}\left|k l a_{i+1}-k l a_{i}\right|
\end{aligned}
$$

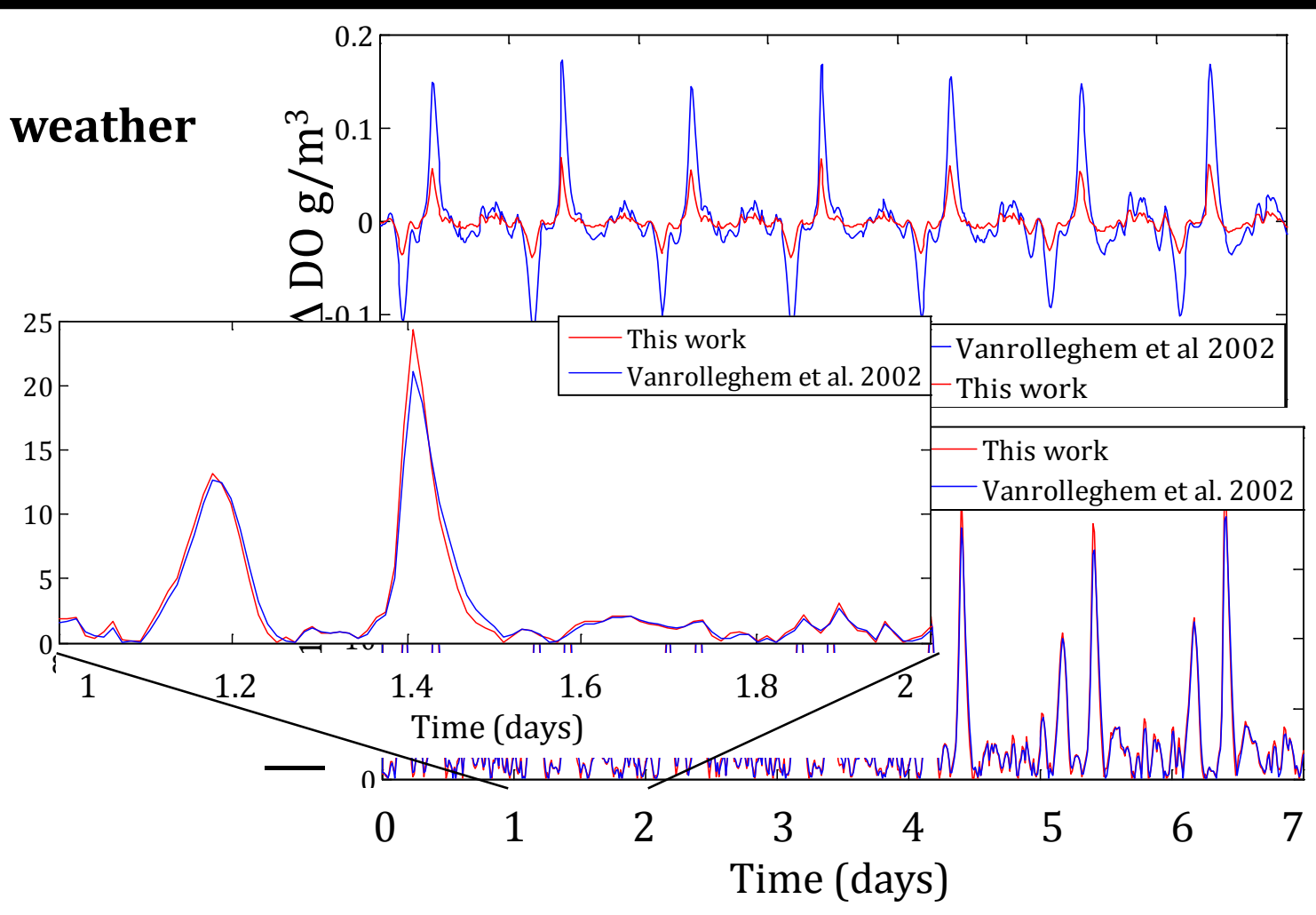

Integral of absolute error $\left(\mathrm{mgO}_{2} \mathrm{~d} \mathrm{~L}^{-1}\right)$

\begin{tabular}{|c|c|c|c|c|c|c|}
\hline & $\mathrm{DO}_{3}$ & $\mathrm{DO}_{4}$ & $\mathrm{DO}_{5}$ & $\mathbf{k L a}_{3}$ & $\mathbf{k L a}_{4}$ & $\mathbf{k L a}_{5}$ \\
\hline \multicolumn{7}{|c|}{ Dryweather influent } \\
\hline Vanrolleghem \&Gillot $_{(2002)}$ & 0.190 & 0.326 & 0.315 & 14.0 & 31.5 & 31.3 \\
\hline This work & 0.020 & 0.034 & 0.032 & 16.1 & 35.1 & 34.7 \\
\hline \multicolumn{7}{|c|}{ Storm weather influent } \\
\hline Vanrolleghem \&Gillot $_{(2002)}$ & 0.193 & 0.313 & 0.323 & 13.2 & 31.5 & 33.3 \\
\hline This work & 0.021 & 0.032 & 0.033 & 15.3 & 31.5 & 33.3 \\
\hline
\end{tabular}

Total Variance of MV ( $\left.\mathbf{d}^{-1}\right)$ 


\section{Evaluation}

7 days dry weather +7 days storm weather

$$
\begin{aligned}
& I A E=\int_{0}^{\infty}\left|D O-D O^{s p}\right| d t \\
& T V=\sum_{i=1}^{\infty}\left|k l a_{i+1}-k l a_{i}\right|
\end{aligned}
$$

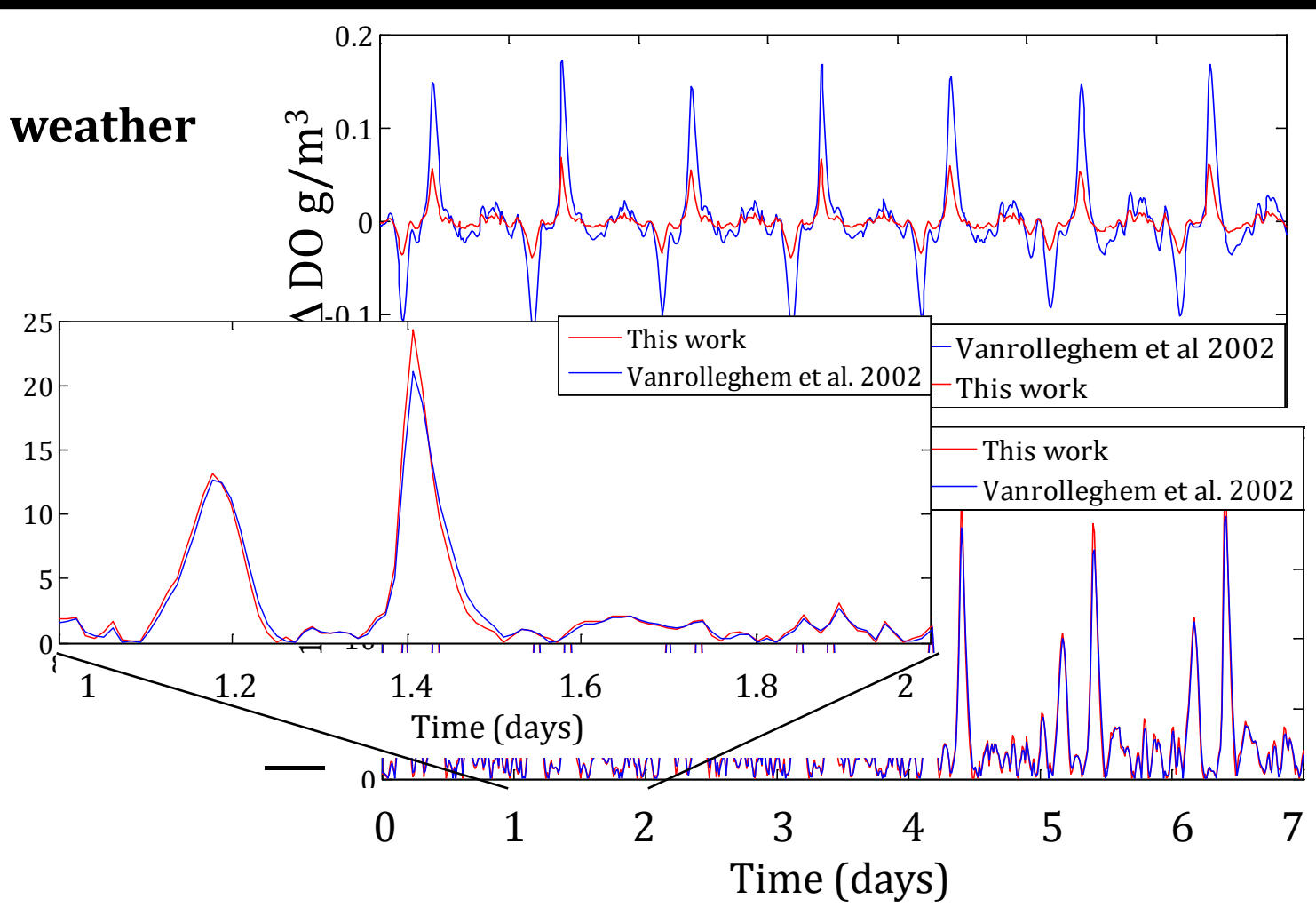

Integral of absolute error $\left(\mathrm{mgO}_{2} \mathrm{~d} \mathrm{~L}^{-1}\right)$

\begin{tabular}{|c|c|c|c|c|c|c|}
\hline & $\mathrm{DO}_{3}$ & $\mathrm{DO}_{4}$ & $\mathrm{DO}_{5}$ & $\mathbf{k L a}_{3}$ & $\mathbf{k L a}_{4}$ & $\mathbf{k L a}_{5}$ \\
\hline \multicolumn{7}{|c|}{ Dryweather influent } \\
\hline Vanrolleghem \&Gillot $_{(2002)}$ & 0.190 & 0.326 & 0.315 & 14.0 & 31.5 & 31.3 \\
\hline This work & 0.020 & 0.034 & 0.032 & 16.1 & 35.1 & 34.7 \\
\hline \multicolumn{7}{|c|}{ Storm weather influent } \\
\hline Vanrolleghem \&Gillot $_{(2002)}$ & 0.193 & 0.313 & 0.323 & 13.2 & 31.5 & 33.3 \\
\hline This work & 0.021 & 0.032 & 0.033 & 15.3 & 31.5 & 33.3 \\
\hline
\end{tabular}

Total Variance of MV ( $\left.\mathbf{d}^{-1}\right)$ 


\section{Conclusions}

We developed a systematic methodology to formally approach the tuning of controllers in WWTP

It takes into account the knowledge of disturbances in WWTP \& user specified performance requirement (DCVs, deltaymax)

Information about the inflow is included to improve the tuning

It requires a model. Which is commonly used in WWTPs process design and operation.

It targets mainly existing plants but can be also used at design and planning stage.

The newly tuned controller showed superior performance

Some upcoming ideas...

Performance evaluation of the control structure including uncertainty, i.e. the combination of sensors and actuators, and how these relate to the control objectives 


\title{
Controller tuning in wastewater treatment plants
}

Funded by Danish Agency for Science, Technology and Innovation through the Research Centre for Design of Microbial Communities in Membrane Bioreactors (09-067230) for funding of the project

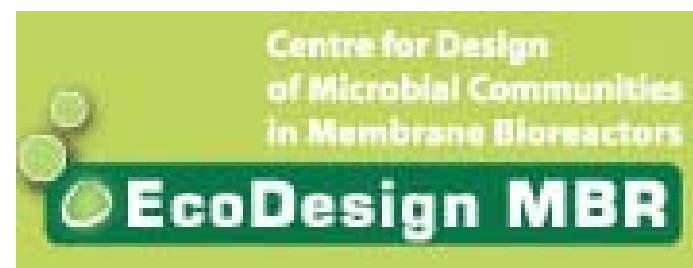

\author{
Miguel Mauricio-I glesias \\ Sten Bay J ørgensen \\ Gürkan Sin \\ mim@kt.dtu.dk
}
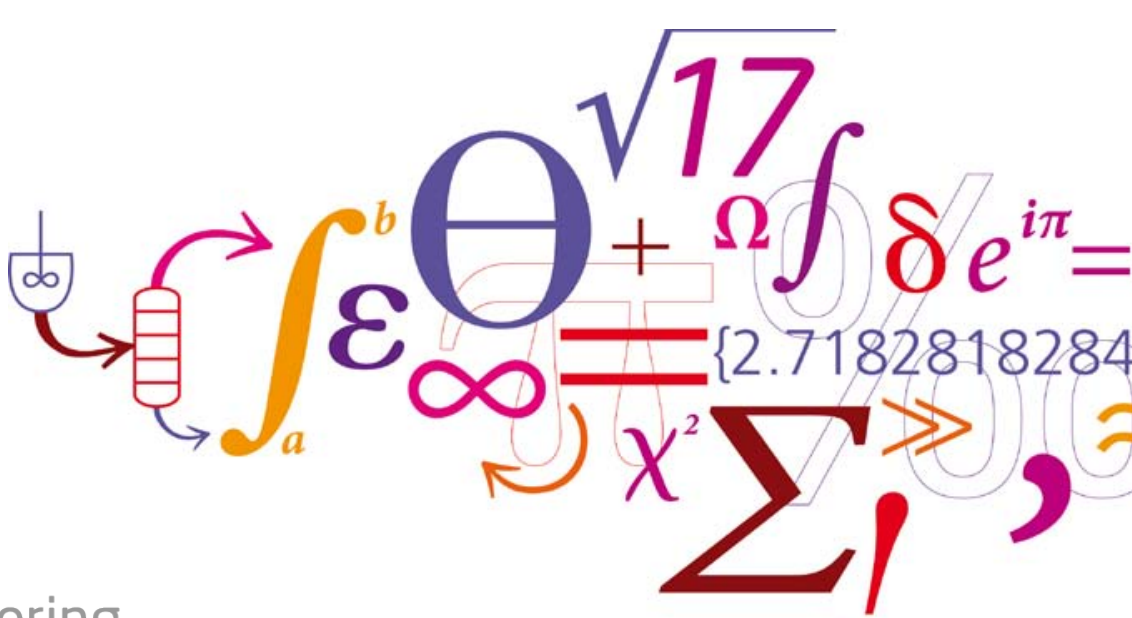